\title{
ON THE DERIVATION OF THE \\ KHOKHLOV-ZABOLOTSKAYA-KUZNETSOV (KZK) EQUATION AND VALIDATION OF THE KZK-APPROXIMATION FOR VISCOUS AND NON-VISCOUS THERMO-ELASTIC MEDIA*
}

\author{
ANNA ROZANOVA-PIERRAT ${ }^{\dagger}$
}

\begin{abstract}
We consider the derivation of the Khokhlov-Zabolotskaya-Kuznetzov (KZK) equation from the nonlinear isentropic Navier-Stokes and Euler systems. The KZK equation is a mathematical model that describes the nonlinear propagation of a finite-amplitude sound pulse in a thermo-viscous medium [S.I. Aanonsen and al., J. Acoust. Soc. Am., 75, 749-768, 1984], [M.A. Averkiou, Y.S. Lee and M.F. Hamilton, J. Acoust. Soc. Am., 94, 2876-2883, 1993], [M.A. Averkiou and M.F. Hamilton, J. Acoust. Soc. Am., 102, 2539-2548, 1997], [A. Kitkauskaite and A. Kopustinskas, available at http://www.etf.rtu.lv/Latvieshu\%20lapa/pasn_str/konf/p_7.pdf], [Y.S. Lee and M.F. Hamilton, J. Acoust. Soc. Am., 97, 906-917, 1995]. The derivation of the KZK equation has to date been based on the paraxial approximation of small perturbations around a given state of the Navier-Stokes system [N.S. Bakhvalov, Ya. M. Zhileikin and E.A. Zabolotskaya, American Institute of Physics, New York, 1987, Nelineinaya teoriya zvukovih puchkov, Moscow "Nauka", 1982]. However, this method does not guarantee that the solution of the initial Navier-Stokes system can be reconstructed from the solution of the KZK equation. We introduce a corrector function in the derivation ansatz that allows one to validate the KZK-approximation. We also give the analysis of other types of derivation [P. Donnat, J.L. Joly, G. Metivier and J. Rauch, Indiana Univ. Math. J., 47, 1167-1241, 1998], [D. Sanchez, J. Diff. Equ., 210, 263-289, 2005], [B. Texier, Adv. Diff. Equ., 9, 1-52, 2004]. We prove the validation of the KZK-approximation for the non-viscous case, as well as for the viscous nonlinear and linear cases. The results are obtained in Sobolev spaces for functions periodic in one of the space variables and with a mean value of zero. The existence of a unique regular solution of the isentropic Navier-Stokes system in the half space with boundary conditions that are both periodic and mean value zero in time is also obtained.
\end{abstract}

Key words. KZK equation, isentropic Navier-Stokes system, entropy, paraxial approximation.

AMS subject classifications. 35Q, 41A65, 58J37, 76N10, 76N99, 76L05.

\section{Introduction}

In this paper we derive the Khokhlov-Zabolotskaya-Kuznetzov (KZK) equation written for the space variables $\left(x_{1}, x^{\prime}\right) \in \mathbb{R}^{n}\left(x_{1} \in \mathbb{R}\right.$ and $\left.x^{\prime} \in \mathbb{R}^{n-1}\right)$ :

$$
c \partial_{\tau z}^{2} I-\frac{(\gamma+1)}{4 \rho_{0}} \partial_{\tau}^{2} I^{2}-\frac{\nu}{2 c^{2} \rho_{0}} \partial_{\tau}^{3} I-\frac{c^{2}}{2} \Delta_{y} I=0
$$

and we validate the KZK-approximation of the Euler $(\nu=0)$ and Navier-Stokes systems $(\nu>0)$. Here $I(\tau, z, y)$ denotes the density of the beam, $t$ is the time, $\tau=t-x_{1} / c$ is the retarded time variable, $c$ is the speed of sound, $z=\epsilon x_{1}$ is the propagation axis, $\epsilon$ is a small enough positive fixed real number, for example, $\epsilon=10^{-5}$ (see section 3), $y=\sqrt{\epsilon} x^{\prime}$ is the transverse axis, $\rho_{0}$ is the density of the medium, $\nu$ is the diffusion constant of the sound for a thermo-viscous fluid or, simply, the viscous coefficient, and $\gamma$ is the adiabatic power.

The KZK Equation (1.1) contains the propagation term $\partial_{\tau z}^{2} I$, the nonlinear term $\partial_{\tau}^{2} I^{2}$, the absorption term $\partial_{\tau}^{3} I$ and the diffractive term $\Delta_{y} I$. The KZK equation was originally derived as a tool for the description of nonlinear acoustic beams $[5,30]$.

\footnotetext{
${ }^{*}$ Received: October 15, 2008; accepted (in revised version): June 25, 2009. Communicated by Francois Golse.

${ }^{\dagger}$ PMC, Laboratoire de Physique de la Matière Condensée, École Polytechnique, France (anna.rozanova-pierrat@polytechnique.edu). http://pmc.polytechnique.fr/ apr/
} 
More precisely, the KZK equation describes acoustic beams for the space variables $\left(x_{1}, x^{\prime}\right)$ with the following properties

1. The beams are concentrated near the $x_{1}$-axis;

2 . The beams propagate along the $x_{1}$-direction;

3. The beams are generated either by an initial condition or by a forcing term on the boundary $x_{1}=0$.

This corresponds to the description of the quasi-one-dimensional propagation of a signal in a homogeneous nonlinear isentropic medium. The KZK equation is applied in acoustical problems as a mathematical model that describes the nonlinear propagation of a finite-amplitude sound beam pulse in the thermo-viscous medium $[1,3,4,14,16]$. It is also used in several other fields, in particular, the description of long waves in ferromagnetic media [25].

The KZK Equation (1.1), as demonstrated (in water and glycerin) in [3], accurately describes the entire process of self-demodulation throughout the near field and into the far field, both on and off the axis of the beam. The use of intense ultrasound in medical and industrial applications has increased considerably in recent years [4]. Both plane and focused sources are used widely in either continuous wave or pulsed mode, and at intensities which lead to nonlinear effects such as harmonic generation and shock formation. Typical ultrasonic sources generate strong diffraction phenomena, which combine with finite amplitude effects to produce waveforms that vary from point to point within the sound beam. Nonlinear effects have become very important at acoustic intensities employed in many current therapeutic and surgical procedures, in particular, in ultrasonic medical imagery, since the human body is a nonlinear medium. In addition, biological media can introduce significant absorption of sound, which must also be considered.

The KZK equation presents a mathematical model of these phenomena. For example, the KZK equation is used in lithotripsy [2, 20, 22]. The principle consists in sending ultrasound waves to break kidney stones (by a mechanical effect). It corresponds to the emission of a very short signal which generates a shock wave. The formation of a shock wave ensures the desired mechanical effect. Another example is the High Intensity Focused Ultrasound Therapy [11, 19, 22, 26]. The goal is to burn tumors in the brain (by a thermal effect). Very long signals are emitted during several seconds. Due to its nonlinearity, the KZK equation accurately models the thermal effect. The next application is "harmonic imagery" $[12,15,31]$. The quadratic nonlinear approach in the high frequency range (harmonics) by the KZK equation permits echographic images of high quality.

Using the results of [24], we study the derivation and approximation of the KZK Equation (1.1) with $I(\tau, z, y)=I\left(t-\frac{x_{1}}{c}, \epsilon x_{1}, \sqrt{\epsilon} x^{\prime}\right), x=\left(x_{1}, x^{\prime}\right)$, where $x_{1} \in \mathbb{R}$ and $x^{\prime} \in \mathbb{R}^{n-1}$. The KZK Equation (1.1) can be obtained from the isentropic Navier-Stokes system for viscous media

$$
\partial_{t} \rho_{\epsilon}+\operatorname{div}\left(\rho_{\epsilon} u_{\epsilon}\right)=0, \quad \rho_{\epsilon}\left[\partial_{t} u_{\epsilon}+\left(u_{\epsilon} \cdot \nabla\right) u_{\epsilon}\right]=-\nabla p\left(\rho_{\epsilon}\right)+\epsilon \nu \Delta u_{\epsilon}
$$

with the approximate state equation for $\rho_{\epsilon}=\rho_{0}+\epsilon \tilde{\rho}_{\epsilon}$, not including terms of the order of $o\left(\epsilon^{2}\right)$,

$$
p=p\left(\rho_{\epsilon}\right)=c^{2} \epsilon \tilde{\rho}_{\epsilon}+\frac{(\gamma-1) c^{2}}{2 \rho_{0}} \epsilon^{2} \tilde{\rho}_{\epsilon}^{2} .
$$

For the non-viscous case $\nu=0$, the KZK Equation (1.1) can be obtained from the isentropic Euler system. 
Let us present the content of the paper. In section 2 we start with a brief summary of mathematical properties of the KZK equation proved in [24] and useful in what follows, and introduce notations.

We consider the derivation of the KZK equation in section 3. Sections 4, 5 contain the validation of the derivation studied for different (viscous, non-viscous, linear and non-linear) cases.

In subsection 3.1, the derivation of the KZK Equation (1.1) from the NavierStokes system (1.2) is taken from the literature [5]. The small perturbations around a given state of the Navier-Stokes system are used with a paraxial approximation, which together compose "the ansatz" reflecting the property that the wave is concentrated near the $x_{1}$-axis.

In subsection 3.2 we explain the limitations of the derivation of the previous subsection. The derivation method of [5] does not ensure that the solution of the initial Navier-Stokes system can be reconstructed by the known solution of the KZK equation. To make it possible moreover with the same order of error as for the derivation, we introduce a Hilbert expansion type construction to the initial ansatz by adding a corrector function of higher order of smallness. This allows one to validate the KZK-approximation.

In section 3.3 we compare this derivation with others from $[8,25,28]$. We analyze the derivation of the KZK equation for the non-viscous case in terms of diffractive nonlinear geometrical optics with rectification [8]. In addition, it will be analyzed in terms of the short wave approximation [28] and the derivation of the ZabolotskayaKhokhlov equation from the Landau-Lifshitz-Maxwell system [25].

In sections 4 and 5 we are interested how the KZK Equation (1.1) provides asymptotic solutions $I(\tau, z, y)=I\left(t-\frac{x_{1}}{c}, \epsilon x_{1}, \sqrt{\epsilon} x^{\prime}\right)$ of system (1.2) denoted $\Phi\left(\rho_{\epsilon}, u_{\epsilon}, \nu\right)=0$, which we call in what follows the exact system.

The viscosity $\nu$ introduces some difference in the construction and we treat separately the cases $\nu=0$ and $\nu \neq 0$. For both cases the ideas of the proofs are based on the convexity of the entropy for the Euler system [6].

With null viscosity both the nonlinear system of elasticity $[6] \Phi\left(\rho_{\epsilon}, u_{\epsilon}, 0\right)=0$ and the KZK equation are well posed (existence and stability of the solutions) for positive and negative but finite time. Consequently, the approximation problem is considered in a time cone. We prove a large time validity of the approximation for non-viscous thermo-elastic media between the KZK solution and the solution of the Euler system in a cone in section 4 .

The viscous case is treated in section 5. If $\nu>0$, we have global existence and stability with small enough initial data for the KZK equation. We also prove (see Theorem 5.5) large time existence (for positive time but under a smallness hypothesis of initial data up to infinity) and stability for the Navier-Stokes system in a half space with a convenient condition on $\rho$. Therefore the approximation is considered in a half space.

However, we first approach the viscous case by a linear problem in order to compare the errors for the linear and nonlinear problems (see Table 5.1). We start with the linear case in subsection 5.1. We establish a large time validity of the approximation for the linear KZK equation

$$
2 c \partial_{\tau z}^{2} I-c^{2} \triangle_{y} I-\frac{\nu}{\rho_{0} c^{2}} \partial_{\tau}^{3} I=0,
$$


and linearized Navier-Stokes system

$$
\begin{aligned}
\partial_{t} \rho+\rho_{0} \nabla \cdot u & =0, \\
\rho_{0} \partial_{t} u+\nabla p(\rho) & =\epsilon \nu \Delta u .
\end{aligned}
$$

To prove the approximation in the non-linear case we need a large-time existence result for a smooth solution of the Navier-Stokes system.

In subsection 5.2, we show the existence of a unique regular solution of the isentropic Navier-Stokes system in the half space with boundary conditions that are periodic and mean value zero in time. Due to the periodicity of the boundary condition in time, it changes sign an infinite number of times. To control the changes in inflow/outflow regions we have to assume that the boundary condition (which is a known function of the initial data for the KZK equation) for fixed $t$ has the same sign for all $x^{\prime} \in \mathbb{R}^{n-1}$, and during one time-period the sign changes only a finite number of times. This restriction avoids a change in the type of the boundary condition in the tangential variables. The typical example of the initial data for the KZK equation which satisfies these conditions is given by Zabolotskaya in [5] by the expression $I(t, 0, y)=-F(y) \sin t$ with $F(y) \geq 0$ (a Gaussian or polynomial beam).

In subsection 5.3, we prove the approximation for viscous thermo-elastic media between the KZK solution and the solution of the Navier-Stokes system in a half space. We obtain as a consequence the validation of the approximation for an admissible weak solution of the Navier-Stokes system satisfying the boundary conditions in the half space. The result is based on the works of $[17,21]$.

In section 6 , we collect the results in the comparative Table 5.1 and give a short conclusion. The announcement of the results can be found in [23].

\section{Preliminaries and notations}

The results of this paper are related to the results of our previous paper [24]. Let us briefly summarize them.

The existence of regular solutions of the KZK equation was studied in [24]:

$$
\left(u_{t}-u u_{x}-\beta u_{x x}\right)_{x}-\gamma \triangle_{y} u=0 \quad \text { in } \quad\left(\mathbb{R}_{x} /(L \mathbb{Z})\right) \times \Omega_{y},
$$

in the class of $L$-periodic functions with respect to the variable $x$ and with mean value zero

$$
\mathcal{D}=\left\{u \in H^{s} \mid u(x+L, y, t)=u(x, y, t), \quad \int_{0}^{L} u(x, y, t) d x=0\right\} .
$$

We suppose in what follows that $\beta$ and $\gamma$ are positive constants. The domain $\Omega_{y}$ is a subset of $\mathbb{R}^{n-1}$ or $\Omega_{y}=\mathbb{R}^{n-1}$. Equation (2.1) can be obtained from equation (1.1) by the following change of notation:

$$
z:=t, \quad \tau:=x, \quad y:=y, \quad I:=\frac{u}{\sqrt{\frac{\gamma+1}{4 c \rho_{0}}}} .
$$

The notation $u \in H^{s}$ means $u(\cdot, \cdot, t) \in H^{s}\left((\mathbb{R} /(L \mathbb{Z})) \times \Omega_{y}\right)$ for $s \geq 0$ and

$$
\|u\|_{H^{s}}=\left(\int_{\Omega_{y}} \sum_{k=-\infty}^{+\infty}\left(1+k^{2}+\eta^{2}\right)^{s}|\hat{u}(k, \eta)|^{2} d \eta\right)^{\frac{1}{2}}<\infty,
$$


where $\hat{u}=\mathcal{F}(u)$ is the Fourier transform of $u$.

The introduction (see $[13,29]$ ) of the operator $\partial_{x}^{-1}: \mathcal{D} \rightarrow \mathcal{D}$ defined by formula

$$
\partial_{x}^{-1} f=\sum_{k \neq 0} \frac{\widehat{f(k)}}{2 \pi i \frac{k}{L}} e^{2 i \pi \frac{k x}{L}}=\int_{0}^{x} f(s) d s+\int_{0}^{L} \frac{s}{L} f(s) d s
$$

allows us to consider the following equivalent equation instead of equation (2.1)

$$
u_{t}-u u_{x}-\beta u_{x x}-\gamma \partial_{x}^{-1} \Delta_{y} u=0 \quad \text { in }\left(\mathbb{R}_{x} /(L \mathbb{Z})\right) \times \Omega_{y} .
$$

The following theorem was proved for this integrated KZK equation.

Theorem 2.1. [24] Consider the Cauchy problem

$$
\left\{\begin{array}{l}
u_{t}-u u_{x}-\beta u_{x x}-\gamma \partial_{x}^{-1}\left(\Delta_{y} u\right)=0 \quad \text { in }\left(\mathbb{R}_{x} /(L \mathbb{Z})\right) \times \mathbb{R}^{n-1}, \\
u(x, y, 0)=u_{0},
\end{array}\right.
$$

in the class $\mathcal{D}$ with the operator $\partial_{x}^{-1}$ defined by formula (2.3) and with $\beta \geq 0$. The following results hold true:

1. For $s>\left[\frac{n}{2}\right]+1$ there exists a constant $C(s, L)$ such that for any initial data $u_{0} \in H^{s}$ on an interval $[0, T[$ with

$$
T \geq \frac{1}{C(s, L)\left\|u_{0}\right\|_{H^{s}}},
$$

problem (2.5) has a unique solution in $C\left(\left[0, T\left[, H^{s}\right) \cap C^{1}\left(\left[0, T\left[, H^{s-2}\right)\right.\right.\right.\right.$.

2. Let $T^{*}$ be the largest time on which such a solution is defined. Then we have

$$
\int_{0}^{T^{*}} \sup _{x, y}\left(\left|\partial_{x} u(x, y, t)\right|+\left|\nabla_{y} u(x, y, t)\right|\right) d t=\infty .
$$

3. If $\beta>0$, we have global existence for small enough initial data: there exists a constant $C_{1}$ such that

$$
\left\|u_{0}\right\|_{H^{s}} \leq C_{1} \Rightarrow T^{*}=\infty .
$$

4. For two solutions $u$ and $v$ of the $K Z K$ equation, assume that $u \in L_{\infty}\left(\left[0, T\left[; H^{s}\right)\right.\right.$ and $v \in L^{2}\left(\left[0, T\left[; L_{2}\right)\right.\right.$. Then we have the following stability and uniqueness result:

$$
\|u(, t)-v(., t)\|_{L^{2}} \leq e^{\int_{0}^{t} s u p_{x, y}\left|\partial_{x} u(x, y, s)\right| d s}\|u(., 0)-v(., 0)\|_{L^{2}} .
$$

REmark 2.2. We note that when $\beta=0$ (i.e., a non-viscous medium), all the corresponding statements of Theorem 2.1 remain valid for $0>t>-C$ with a suitable $C$.

In what follows we use the global existence in time of the KZK solution for small enough initial data if $\beta>0$ (see point 3 of Theorem 2.1) and the blow-up result (existence of a shock wave) if $\beta=0$. Indeed, when $\gamma=0$ and $\beta>0$, equation (2.1) reduces to the Burgers-Hopf equation for which the existence, smoothness, and uniqueness of the solution are well known. Furthermore, for $\gamma=\beta=0$ it reduces to the Burgers' equation

$$
\partial_{t} u-\partial_{x} \frac{u^{2}}{2}=0,
$$


which, after a finite time, exhibits singularities. Specifically, the following theorem was proved in [24]:

Theorem 2.3. [24] The equation

$$
\left(u_{t}-u u_{x}\right)_{x}-\gamma \Delta_{y} u=0 \quad \text { in } \quad \mathbb{R}_{t_{+}} \times \mathbb{R}_{x} \times \Omega_{y}
$$

with a Neumann boundary condition on $\partial \Omega_{y}$ has no smooth global solution in time if

$$
\sup _{x, y} \partial_{x} u(x, y, 0)
$$

is large enough with respect to $\gamma$.

Between these two extreme cases of global existence and blow-up there is a "quasishock" wave phenomenon corresponding to the case when $\beta \rightarrow 0$. The existence of a shock wave in the case of propagation of a beam in non-dissipative media and the existence of a quasi-shock wave for dissipative media are obtained numerically in [5]. The latter phenomenon corresponds to the approximation of the shock wave by the front of the beam given by the (smooth) solution which is tentatively global. This phenomenon appears for the KZK equation with a small viscosity coefficient. Following [5], the wave is named a quasi-shock wave if the width of the wave front $\triangle \tau \leq \pi / 10$. Theorem 2.1 and Theorem 2.3 confirm the numerical results. In particular, we observe from [5, pp.78-81] that as $\beta$ becomes smaller $(\beta \rightarrow 0)$, the KZK equation exhibits a quasi-shock which approaches a shock wave, into which the quasi-shock degenerates at $\beta=0$.

Let us introduce some notations used in what follows in this paper. The operator $\nabla$. denotes the divergence operator. For a positive fixed small enough real number $\epsilon$, (see section 3 ), we suppose that $\mathbb{R}_{+}$consists of classes, which are characterized by the power of $\epsilon$ :

$$
\ldots, \epsilon^{\alpha}, \ldots, \epsilon^{2}, \ldots, \epsilon, \ldots, \sqrt{\epsilon}, \ldots, \epsilon^{0}=1, \ldots, \frac{1}{\epsilon}, \ldots, \frac{1}{\epsilon^{2}}, \ldots, \frac{1}{\epsilon^{\alpha}}, \ldots,
$$

where $\alpha>2$ is a rational number. $O(1)$ denotes the class of constants. If $U$ is a matrix, $U^{T}$ is the transpose of $U$.

\section{On the derivation of the KZK equation}

In the present section the emphasis is focused on the derivation of the KZK Equation (1.1) in nonlinear media using the following acoustic properties of beam propagation

1. The beams are concentrated near the $x_{1}$-axis;

2 . The beams propagate along the $x_{1}$-direction;

3. The beams are generated either by an initial condition or by a forcing term on the boundary $x_{1}=0$.

It is assumed that the variation of beam's propagation in the direction

$$
x^{\prime}=\left(x_{2}, x_{3}, \ldots, x_{n}\right)
$$

perpendicular to the $x_{1}$-axis is much larger than its variation along the $x_{1}$-axis, i.e., we suppose that the beam has the form $U\left(t-x_{1} / c, \epsilon x_{1}, \sqrt{\epsilon} x^{\prime}\right)$. The first argument $t-x_{1} / c$ describes the wave propagation in time along the $x_{1}$-axis with the sound speed $c$, and the two last arguments $\epsilon x_{1}$ and $\sqrt{\epsilon} x^{\prime}$ describe the speed of the deformation of the 
wave along the $x_{1}$-axis and along the $x^{\prime}$-axis, respectively. We remark that $\epsilon \ll 1$ and consequently $\epsilon \ll \sqrt{\epsilon}$.

For instance, for the linear wave equation in $\mathbb{R}^{n}(n>1)$ :

$$
\frac{1}{c^{2}} \partial_{t}^{2} u-\Delta u=0
$$

the following ansatz

$$
u_{\epsilon}=U\left(t-\frac{x_{1}}{c}, \epsilon x_{1}, \sqrt{\epsilon} x^{\prime}\right)
$$

containing a "profile" $U(\tau, z, y)$ (with small $\epsilon$ ) leads to the formula:

$$
\partial_{\tau, z}^{2} U-\frac{c}{2} \Delta_{y} U=O(\epsilon)
$$

or for functions $U(\tau, z, y)=A(z, y) e^{i \omega \tau}$, to the equation

$$
i \omega \partial_{z} A-\frac{c}{2} \Delta_{y} A=O(\epsilon)
$$

We observe that with $\epsilon=0$ equations (3.3) and (3.4) are two variants of the classical paraxial approximation and that equation (3.3) contains the linear non-diffusive terms of the KZK equation which usually has the following form for some positive constants $\beta$ and $\gamma$ :

$$
\partial_{\tau, z}^{2} U-\frac{1}{2} \partial_{\tau}^{2} U^{2}-\beta \partial_{\tau}^{3} U-\gamma \Delta_{y} U=0 .
$$

Conversely, the isentropic evolution of the thermo-elastic non-viscous media is given by the following Euler system

$$
\partial_{t} \rho+\operatorname{div}(\rho v)=0, \quad \rho\left(\partial_{t} v+v \cdot \nabla v\right)=-\nabla p(\rho) .
$$

Any constant state $\left(\rho_{0}, v_{0}\right)$ is a stationary solution of system (3.5). Linearization near this state introduces the variables

$$
\rho=\rho_{0}+\epsilon \tilde{\rho}, \quad v=v_{0}+\epsilon \tilde{v}
$$

and for $v_{0}=0$ the acoustic system:

$$
\partial_{t} \tilde{\rho}+\rho_{0} \nabla \cdot \tilde{v}=0, \quad \rho_{0} \partial_{t} \tilde{v}+p^{\prime}\left(\rho_{0}\right) \nabla \tilde{\rho}=0 .
$$

System (3.6) is equivalent to the wave equation:

$$
\frac{1}{c^{2}} \partial_{t}^{2} \tilde{\rho}-\Delta \tilde{\rho}=0, \quad \partial_{t} \tilde{v}=-\frac{p^{\prime}\left(\rho_{0}\right)}{\rho_{0}} \nabla \tilde{\rho},
$$

where $c=\sqrt{p^{\prime}\left(\rho_{0}\right)}$ is the speed of sound in the unperturbed media.

We observe that equation (3.3), which is the linearized and non-viscous part of the KZK equation, can be obtained in two steps. First we consider small perturbations around a constant state of the isentropic Euler system which are solutions to the acoustic equation, and then we consider a paraxial approximation of such solutions.

The derivation of the complete KZK equation follows almost the same line. It takes into account the viscosity and the size of the nonlinear terms. 
3.1. Standard physical derivation [5]. We start from the Navier-Stokes system:

$$
\partial_{t} \rho+\operatorname{div}(\rho u)=0, \quad \rho\left[\partial_{t} u+(u \cdot \nabla) u\right]=-\nabla p(\rho, S)+b \Delta u,
$$

where $S$ is the entropy and the state law $p=p(\rho, S)$ is the pressure.

First, we assume that the temperature $T$ and the entropy $S$ have small increments $\tilde{T}$ and $\tilde{S}$. With the hypothesis of potential motion, we introduce constant states

$$
\rho=\rho_{0}, \quad u=u_{0} .
$$

Next, we assume that the density fluctuation (around the constant state $\rho_{0}$ ) and the velocity fluctuation (around $u_{0}$, which can be taken equal to zero using a Galilean transformation), are of the same order of $\epsilon$ :

$$
\rho_{\epsilon}=\rho_{0}+\epsilon \tilde{\rho}_{\epsilon}, \quad u_{\epsilon}=\epsilon \tilde{u}_{\epsilon},
$$

where $\epsilon$ is a dimensionless parameter which characterizes the smallness of the perturbation. For instance, in water with an initial power of the order of $0.3 \mathrm{~W} / \mathrm{cm}^{2} \epsilon$ is equal to $10^{-5}$. We also suppose that the viscosity coefficient is small:

$$
b=\epsilon \tilde{b} \text {. }
$$

Using the transport heat equation in the form

$$
\rho_{0} T_{0} \partial_{t} \tilde{S}=\kappa \triangle \tilde{T}
$$

where $\kappa$ is a dissipation coefficient, the approximate state equation

$$
p=c^{2} \epsilon \tilde{\rho}_{\epsilon}+\frac{1}{2}\left(\partial_{\rho}^{2} p\right)_{S} \epsilon^{2} \tilde{\rho}_{\epsilon}^{2}+\left(\partial_{S} p\right)_{\rho} \tilde{S}
$$

(where the notation $(\cdot)_{S}$ means that the expression in brackets is constant in $S$ ) can be replaced [5], due to the following relation:

$$
\tilde{S}=-\frac{\kappa}{T_{0}}\left(\partial_{p} T\right)_{S} \operatorname{div} u_{\epsilon},
$$

by

$$
p=c^{2} \epsilon \tilde{\rho}_{\epsilon}+\frac{(\gamma-1) c^{2}}{2 \rho_{0}} \epsilon^{2} \tilde{\rho}_{\epsilon}^{2}-\kappa\left(\frac{1}{C_{v}}-\frac{1}{C_{p}}\right) \nabla \cdot u_{\epsilon}
$$

Here $\gamma=C_{p} / C_{v}$ denotes the ratio of the heat capacities at constant pressure and at constant volume respectively. System (3.8) becomes an isentropic system

$$
\partial_{t} \rho_{\epsilon}+\operatorname{div}\left(\rho_{\epsilon} u_{\epsilon}\right)=0, \quad \rho\left[\partial_{t} u_{\epsilon}+\left(u_{\epsilon} \cdot \nabla\right) u_{\epsilon}\right]=-\nabla p\left(\rho_{\epsilon}\right)+\epsilon \nu \Delta u_{\epsilon},
$$

with the approximate state equation (see equation (1.3))

$$
p=p\left(\rho_{\epsilon}\right)=c^{2} \epsilon \tilde{\rho}_{\epsilon}+\frac{(\gamma-1) c^{2}}{2 \rho_{0}} \epsilon^{2} \tilde{\rho}_{\epsilon}^{2}
$$

and with a small enough and positive viscosity coefficient:

$$
\epsilon \nu=b+\kappa\left(\frac{1}{C_{v}}-\frac{1}{C_{p}}\right) .
$$


Next, we recall the direction of propagation of the beam, along the $x_{1}$-axis, and therefore consider the following profiles:

$$
\begin{aligned}
& \tilde{\rho}_{\epsilon}\left(x_{1}, x^{\prime}, t\right)=I\left(t-\frac{x_{1}}{c}, \epsilon x_{1}, \sqrt{\epsilon} x^{\prime}\right) \\
& \tilde{u}_{\epsilon}\left(x_{1}, x^{\prime}, t\right)=\left(u_{\epsilon, 1}, u_{\epsilon}^{\prime}\right)=\left(v\left(t-\frac{x_{1}}{c}, \epsilon x_{1}, \sqrt{\epsilon} x^{\prime}\right), \sqrt{\epsilon} \vec{w}\left(t-\frac{x_{1}}{c}, \epsilon x_{1}, \sqrt{\epsilon} x^{\prime}\right)\right) .
\end{aligned}
$$

We suppose with the help of Equation (3.13) that the velocity $u_{\epsilon}\left(x_{1}, x^{\prime}, t\right)$ being a vector function has two different perturbations along the $x_{1}$-axis and $x^{\prime}$-axis: the perturbation $\epsilon v$ in the $x_{1}$-direction and the perturbation $\epsilon^{\frac{3}{2}} \vec{w}$ in the $x^{\prime}$-direction. In equations (3.12) and (3.13) the argument of the functions will be denoted by $(\tau, z, y)$ and $c$ is the speed of sound, i.e., $c=\sqrt{p^{\prime}\left(\rho_{0}\right)}$. Inserting the functions $\rho_{\epsilon}=\rho_{0}+\epsilon I$ and $u_{\epsilon}=\epsilon(v, \sqrt{\epsilon} \vec{w})$ into system (3.10), we obtain the following.

1. For mass conservation,

$$
\begin{aligned}
& \partial_{t} \rho_{\epsilon}+\operatorname{div}\left(\rho_{\epsilon} u_{\epsilon}\right)=\epsilon\left(\partial_{\tau} I-\frac{\rho_{0}}{c} \partial_{\tau} v\right) \\
& +\epsilon^{2}\left(\rho_{0}\left(\partial_{z} v+\nabla_{y} \cdot \vec{w}\right)-\frac{1}{c} v \partial_{\tau} I-\frac{1}{c} I \partial_{\tau} v\right)+O\left(\epsilon^{3}\right)=0 .
\end{aligned}
$$

2. For momentum conservation in the $x_{1}$-direction,

$$
\begin{aligned}
& \rho_{\epsilon} \epsilon\left(\partial_{t} u_{\epsilon, 1}+u_{\epsilon} \cdot \nabla u_{\epsilon, 1}\right)+\partial_{x_{1}} p\left(\rho_{\epsilon}\right)-\epsilon^{2} \nu \Delta u_{\epsilon, 1}=\epsilon\left(\rho_{0} \partial_{\tau} v-c \partial_{\tau} I\right) \\
& +\epsilon^{2}\left(I \partial_{\tau} v-\frac{\rho_{0}}{c} v \partial_{\tau} v+c^{2} \partial_{z} I-\frac{(\gamma-1)}{2 \rho_{0}} c \partial_{\tau} I^{2}-\frac{\nu}{c^{2}} \partial_{\tau}^{2} v\right)+O\left(\epsilon^{3}\right)=0
\end{aligned}
$$

3. For the orthogonal (to the $x_{1}$-axis) component of the momentum,

$$
\begin{aligned}
& \rho_{\epsilon} \epsilon\left(\partial_{t} u_{\epsilon}^{\prime}+u_{\epsilon} \cdot \nabla \cdot u_{\epsilon}^{\prime}\right)+\partial_{x^{\prime}} p\left(\rho_{\epsilon}\right)-\epsilon^{2} \nu \Delta u_{\epsilon}^{\prime}=\epsilon^{\frac{3}{2}}\left(\rho_{0} \partial_{\tau} \vec{w}+c^{2} \nabla_{y} I\right) \\
& +\epsilon^{\frac{5}{2}}\left(-\frac{\rho_{0} v}{c} \partial_{\tau} \vec{w}+I \partial_{\tau} \vec{w}+\frac{(\gamma-1) c^{2}}{2 \rho_{0}} \nabla_{y} I^{2}-\frac{\nu}{c^{2}} \Delta_{y} \vec{w}\right)+O\left(\epsilon^{3}\right)=0 .
\end{aligned}
$$

To eliminate the terms of the first and second order in $\epsilon$ we need to pose:

$$
\begin{aligned}
\partial_{\tau} I-\frac{\rho_{0}}{c} \partial_{\tau} v & =0 \\
\rho_{0}\left(\partial_{z} v+\nabla_{y} \cdot \vec{w}\right)-\frac{1}{c} v \partial_{\tau} I-\frac{1}{c} I \partial_{\tau} v & =0 \\
I \partial_{\tau} v-\frac{\rho_{0}}{c} v \partial_{\tau} v+c^{2} \partial_{z} I-\frac{(\gamma-1)}{2 \rho_{0}} c \partial_{\tau} I^{2}-\frac{\nu}{c^{2}} \partial_{\tau}^{2} v & =0
\end{aligned}
$$

Therefore, $I$ and $v$ should be linked by the formula:

$$
v=\frac{c}{\rho_{0}} I
$$

and the second order terms of equations (3.14) and (3.15) by the formula:

$$
\begin{aligned}
& \rho_{0}\left(\partial_{z} v+\nabla_{y} \cdot \vec{w}\right)-\frac{1}{c} v \partial_{\tau} I-\frac{1}{c} I \partial_{\tau} v \\
= & -\frac{1}{c}\left(I \partial_{\tau} v-\frac{\rho_{0}}{c} v \partial_{\tau} v+c^{2} \partial_{z} I-\frac{(\gamma-1)}{2 \rho_{0}} c \partial_{\tau} I^{2}-\frac{\nu}{c^{2}} \partial_{\tau}^{2} v\right) .
\end{aligned}
$$


Thus (with equation (3.20)), it gives:

$$
\rho_{0} \nabla_{y} \cdot \vec{w}+2 c \partial_{z} I-\frac{(\gamma+1)}{2 \rho_{0}} \partial_{\tau} I^{2}-\frac{\nu}{c^{2} \rho_{0}} \partial_{\tau}^{2} I=0 .
$$

Eventually, we use the equation of the orthogonal moment (3.16) to eliminate the term $\rho_{0} \nabla_{y} \cdot \vec{w}$. Assuming, in agreement with equation (3.16), that

$$
\rho_{0} \partial_{\tau} \vec{w}+c^{2} \nabla_{y} I=0,
$$

and taking the divergence with respect to $y$ of this equation, differentiating equation (3.22) with respect to $\tau$, we obtain

$$
c \partial_{\tau z}^{2} I-\frac{(\gamma+1)}{4 \rho_{0}} \partial_{\tau}^{2} I^{2}-\frac{\nu}{2 c^{2} \rho_{0}} \partial_{\tau}^{3} I-\frac{c^{2}}{2} \Delta_{y} I=0 .
$$

The KZK Equation (3.24) is written for the density perturbation, but the same equation with different constants can also be derived for the pressure and velocity. The transposition of these KZK equations is possible due to relations (3.11), (3.20), and (3.23). For example the equation on the pressure has the form

$$
\partial_{\tau z}^{2} p-\frac{\beta}{2 \rho_{0} c^{3}} \partial_{\tau}^{2} p^{2}-\frac{\delta}{2 c^{3}} \partial_{\tau}^{3} p-\frac{c}{2} \triangle_{y} p=0,
$$

where $\delta$ is the diffusivity constant of the sound for thermo-viscous media and $\beta$ is the nonlinearity constant.

3.2. Introduction of a corrector. The above derivation is standard in physics articles, however, it does not imply that the functions

$$
\rho_{\epsilon}=\rho_{0}+\epsilon I, \quad u_{\epsilon}=\epsilon(v, \sqrt{\epsilon} \vec{w})
$$

are a solution of system (3.10) with an error term of the order of $\epsilon^{3}$. Indeed, we can assume (3.17) and that (3.23) with (3.22) take place, but not the fact that the quantity which corresponds to the term of the order of $\epsilon^{2}$ (both in the mass and momentum conservation along the $x_{1}$-axis) is zero (see (3.18) and (3.19)).

To remedy this fact, and also ensure an error of the order of $\epsilon^{\frac{5}{2}}$ in the equation for the orthogonal momentum to the $x_{1}$-direction, we introduce a Hilbert expansion type construction and write

$$
\rho_{\epsilon}=\rho_{0}+\epsilon I, \quad u_{\epsilon}=\epsilon\left(v+\epsilon v_{1}, \sqrt{\epsilon} \vec{w}\right) .
$$

In (3.25) we assume that $I$ is a solution of the KZK Equation (3.24), while $v$ and $w$ are given in terms of $I$ by equations (3.20) and (3.23). We obtain modulo terms of the order of $\epsilon^{\frac{5}{2}}$, for the right hand side of equations (3.14) and (3.15):

$$
\begin{aligned}
& \epsilon^{2}\left(-\frac{\rho_{0}}{c} \partial_{\tau} v_{1}+\rho_{0}\left(\partial_{z} v+\nabla_{y} \cdot w\right)-\frac{1}{\rho_{0}} \partial_{\tau} I^{2}\right), \\
& \epsilon^{2}\left(\rho_{0} \partial_{\tau} v_{1}+c^{2} \partial_{z} I-\frac{\gamma-1}{2 \rho_{0}} c \partial_{\tau} I^{2}-\frac{\nu}{c \rho_{0}} \partial_{\tau}^{2} I\right) .
\end{aligned}
$$

Taking into account the KZK equation, this implies for the "corrector" $v_{1}$ the relation

$$
\partial_{\tau} v_{1}=\frac{\gamma-1}{2 \rho_{0}^{2}} c \partial_{\tau} I^{2}+\frac{\nu}{c \rho_{0}^{2}} \partial_{\tau}^{2} I-\frac{c^{2}}{\rho_{0}} \partial_{z} I .
$$


The inverse is also true: inserting (3.25) into the Navier-Stokes system (3.10) we find that the perturbation of the density $I$ satisfies the KZK equation. More precisely, using (3.25) we obtain (the operator $\partial_{\tau}^{-1}$ is defined in equation (2.3))

$$
\begin{aligned}
\epsilon\left(\rho_{0} v-c I\right)= & \Rightarrow v(\tau, z, y)=\frac{c}{\rho_{0}} I(\tau, z, y) \\
\epsilon^{\frac{3}{2}}\left(\rho_{0} \partial_{\tau} \vec{w}+c^{2} \nabla_{y} I\right)= & 0 \Rightarrow \\
\vec{w}(\tau, z, y)= & \frac{c^{2}}{\rho_{0}}\left(\int_{0}^{\tau} \nabla_{y} I(s, z, y) d s+\int_{0}^{L} \frac{s}{L} \nabla_{y} I(s, z, y) d s\right) \\
& \epsilon^{2}\left(\rho_{0} \partial_{\tau} v_{1}+c^{2} \partial_{z} I-\frac{\gamma-1}{2 \rho_{0}} c \partial_{\tau} I^{2}-\frac{\nu}{c \rho_{0}} \partial_{\tau}^{2} I\right)=0 \Rightarrow \\
v_{1}(\tau, z, y)= & \frac{c^{3}}{2 \rho_{0}} \partial_{\tau}^{-2} \triangle_{y} I+\frac{(\gamma-1)}{4 \rho_{0}^{2}} c I^{2}-\frac{c(\gamma-1)}{4 L \rho_{0}^{2}} \int_{0}^{L} I^{2} d \tau+\frac{3 \nu}{2 c \rho_{0}^{2}} \partial_{\tau} I \\
& \epsilon^{2}\left(c \partial_{\tau z}^{2} I-\frac{(\gamma+1)}{4 \rho_{0}} \partial_{\tau}^{2} I^{2}-\frac{\nu}{2 c^{2} \rho_{0}} \partial_{\tau}^{3} I-\frac{c^{2}}{2} \Delta_{y} I\right)=0
\end{aligned}
$$

where equation (3.30) is the KZK equation.

3.3. Comparison with other derivations. It is interesting to notice that for the non-viscous case (i.e., for the isentropic compressible Euler system) the KZKlike equation with $\nu=0$ has been obtained using the scaling of nonlinear diffractive geometrical optics with rectification in [8, p. 1233] (in two dimensions). The initial goal of the article $[8]$ is to construct the nonlinear symmetric hyperbolic equation

$$
L\left(u, \partial_{x}\right) u+F(u)=0 .
$$

The case of the isentropic compressible Euler system is given as an example. The basic ansatz in [8] has three scales

$$
u_{\epsilon}(x)=\epsilon^{2} a\left(\epsilon, \epsilon x, x, \frac{x \cdot \beta}{\epsilon}\right),
$$

where

$$
a(\epsilon, X, x, \theta)=a_{0}(X, x, \theta)+\epsilon a_{1}(X, x, \theta)+\epsilon^{2} a_{2}(X, x, \theta) .
$$

Here $x=(t, y) \in \mathbb{R}^{1+d}, \beta=(\tau, \eta) \in \mathbb{R}^{1+d}$ (where $d$ is the dimension size), and the profiles $a_{j}(X, x, \theta)$ are periodic in $\theta$. The KZK-like equation of the form

$$
\partial_{T} a-\triangle_{y} \partial_{\theta}^{-1} a+\sigma a \partial_{\theta} a=0,
$$

with $\sigma \in \mathbb{R}$ determined from an identity and with $T=\frac{t}{\epsilon}$, holds for the profile $a_{0}$ with mean value zero on $\theta$ (for the proof see [8, pp. 1231, 1234]). The property of the profile $a_{0}$ to have mean value zero on $\theta$ corresponds to the vanishing of the non-oscillatory part: with notations of $\left[8\right.$, p.1181], $\underline{a}:=\frac{1}{2 \pi} \int_{0}^{2 \pi} a d \theta$ and the oscillating part is denoted by $a^{*}:=a-\underline{a}$.

An analogous technique is used in [28] to study the short wave approximation for general symmetric hyperbolic systems as

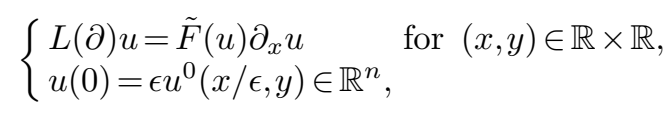


with a hyperbolic operator $L(\partial)=\partial_{t}+A \partial_{x}+B \partial_{y}+E$. "Short waves" stand for shortwavelength approximate solutions, or equivalently, approximate solutions with initial data whose oscillatory frequencies are large compared to the parameters of the system. For the variables

$$
T=\frac{t}{\epsilon}, \quad X=\frac{x}{\epsilon}, \quad y, \quad \tau=\epsilon t
$$

in [28] Texier looks for the approximate solutions in the form

$$
u^{\epsilon}(t, x, y)=\epsilon\left(u_{0}+\epsilon u_{1}+\epsilon^{2} u_{2}\right)(T, X, y, \tau) .
$$

For the first profile $u_{0}$ Texier has a system of the form

$$
\left\{\begin{array}{l}
\left(\partial_{T}+c \partial_{X}\right) u_{0}=0 \\
\left(\partial_{\tau} \partial_{X}-\partial_{Y}^{2}\right) u_{0}=\partial_{X}\left(u_{0} \partial_{X} u_{0}\right)
\end{array}\right.
$$

corresponding to the KZK equation for the function $\tilde{u}_{0}(X-c T, \tau, y, X)$. The estimate of the approximate result in [28] between the exact solution $v^{\epsilon}$ of system (3.31) and the solution $u_{0}^{\epsilon}$ of a system of form (3.33) is the following

$$
\frac{1}{\epsilon}\left\|v^{\epsilon}-\epsilon u_{0}^{\epsilon}\right\|_{L_{\infty}\left(\left[0, \tau_{0} / \epsilon\right] \times \mathbb{R}_{x, y}^{2}\right)}=o(1) .
$$

To analyze common points between this work and the KZK-approximation we can pass from the variables corresponding to our scaling

$$
\left(t-\frac{x_{1}}{c}, \epsilon x_{1}, \sqrt{\epsilon} x^{\prime}\right)
$$

to "variable $=\sqrt{\epsilon}$ variable" in the following way:

$$
\left(\frac{1}{\sqrt{\epsilon}}\left(\tilde{t}-\frac{\tilde{x}_{1}}{c}\right), \sqrt{\epsilon} \tilde{x}_{1}, \tilde{x}^{\prime}\right) .
$$

Supposing now that $\epsilon=\tilde{\epsilon}^{2}$, we obtain

$$
\left(\frac{1}{\tilde{\epsilon}}\left(\tilde{t}-\frac{\tilde{x}_{1}}{c}\right), \tilde{\epsilon} \tilde{x}_{1}, \tilde{x}^{\prime}\right)
$$

and similarly

$$
(\rho, u)=\left(\rho_{0}+\tilde{\epsilon} \tilde{I}, \tilde{\epsilon}\left(\tilde{v}+\tilde{\epsilon}^{2} \tilde{v}_{1}, \tilde{\epsilon} \tilde{w}\right)\right) .
$$

These variables exactly correspond to Texier's case [28]

$$
\left(T-\frac{X}{c}, \tau, y\right)
$$

The first profile $\epsilon u_{0}$ from [28] corresponds to $\left(\rho_{0}+\tilde{\epsilon} \tilde{I}, \tilde{\epsilon} \tilde{v}\right)$ for which we have exactly system (3.33) in the form of equation (3.17) and the KZK equation without the viscous term. The profile $\tilde{\epsilon}^{2} \tilde{w}$ is associated with $\epsilon^{2} u_{1}$ and the profile $\tilde{\epsilon}^{3} \tilde{v}_{1}$ is associated with $\epsilon^{3} u_{2}$. The result of [28] is obtained for the non-periodic case and without the vanishing mean condition, which is important for physical reasons. 
This analysis of the works $[8,28]$ shows that our approach is similar where the variables have been switched with $\epsilon$ "variable $=\sqrt{\epsilon}$ variable" to balance the oscillation,

$$
\frac{1}{\sqrt{\epsilon}}\left(\tilde{t}-\frac{\tilde{x}_{1}}{c}\right) \mapsto\left(t-\frac{x_{1}}{c}\right) .
$$

In other words we can say that we have $O(1)$ oscillations.

The scaling used by Sanchez [25] for the Landau-Lifshitz-Maxwell equations in $\mathbb{R}^{3}$ is very different. Sanchez starts with the system

$$
\begin{aligned}
& \partial_{t} M=-M \wedge H-\frac{\gamma}{|M|} M \wedge(M \wedge H) \text { in } \mathbb{R}^{3}, \\
& \partial_{t}(H+M)-\nabla \wedge E=0 \text { in } \mathbb{R}^{3}, \\
& \partial_{t} E+\nabla \wedge H=0 \text { in } \mathbb{R}^{3},
\end{aligned}
$$

which represents the Landau-Lifshitz-Maxwell equations and admits stable equilibrium solutions where the magnetization $M$ is uniform and everywhere parallel to the effective magnetic field $H$ :

$$
(M, H, E)_{\alpha}=\left(M_{0}, \alpha^{-1} M_{0}, 0\right), \alpha>0,
$$

where $E$ is the electric field. Then Sanchez considers small perturbations of the equilibrium states whose size is measured by a small parameter $\epsilon$. The perturbation is taken in the form:

$$
\begin{aligned}
& M(t, x, y)=M_{0}+\epsilon^{2} \widetilde{M}(\tilde{t}, \tau, \tilde{x}, \tilde{y}), \\
& H(t, x, y)=\alpha^{-1} M_{0}+\epsilon^{2} \widetilde{H}(\tilde{t}, \tau, \tilde{x}, \tilde{y}), \\
& E(t, x, y)=\epsilon^{2} \widetilde{E}(\tilde{t}, \tau, \tilde{x}, \tilde{y}),
\end{aligned}
$$

where $\tilde{t}, \tau, \tilde{x}, \tilde{y}$ are new rescaled variables:

$$
\tilde{t}=\epsilon^{2} t, \quad \tau=\epsilon^{4} t, \quad \tilde{x}=\epsilon^{2} x, \quad \tilde{y}=\epsilon^{3} y .
$$

We notice here that if we take

$$
\hat{t}=\epsilon^{3} t, \quad \hat{x}=\epsilon^{3} x, \quad \hat{y}=\epsilon^{3} y,
$$

we exactly obtain (3.32) from (3.38):

$$
\tilde{t}=\frac{\hat{t}}{\epsilon}, \quad \tau=\epsilon \hat{t}, \quad \tilde{x}=\frac{\hat{x}}{\epsilon}, \quad \tilde{y}=\hat{y} .
$$

But the smallness of functions' profiles is different. The vector of the perturbation $U(\tilde{t}, \tau, \tilde{x}, \tilde{y})=\left(\alpha^{-\frac{1}{2}} \widetilde{M}, \widetilde{H}, \widetilde{E}\right)^{t}(\tilde{t}, \tau, \tilde{x}, \tilde{y})$ satisfies an equation in the form (the $\sim$ on the variables is omitted)

$$
\begin{aligned}
\partial_{t} U+\epsilon^{2} \partial_{\tau} U+A_{1} \partial_{x} U+\epsilon A_{2} \partial_{y} U+\epsilon^{-2} L U & =B(U, U)+\epsilon^{2} T(U, U, U), \\
U(0,0) & =U_{0}^{0},
\end{aligned}
$$

where $A_{1}, A_{2}$, and $L$ are linear operators in $\mathbb{R}^{9}, B$ is a bilinear map on $\mathbb{R}^{9} \times \mathbb{R}^{9}$, and $T$ is a trilinear map on $\mathbb{R}^{9} \times \mathbb{R}^{9} \times \mathbb{R}^{9}$. Using an asymptotic formal expansion of $U$ in 
the form $U \equiv U_{0}+\epsilon U_{1}+\epsilon^{2} U_{2}+\ldots$, Sanchez obtains that the leading term $U_{0}$ breaks down in five traveling and standing terms, $U_{0}=\sum_{j=1}^{5} u_{j}$, which satisfy the transport equation and the Zabolotskaya-Khokhlov equation,

$$
\begin{aligned}
\left(\partial_{t}+v_{j} \partial_{x}\right) u_{j} & =0, \\
\partial_{x}\left(\partial_{\tau} u_{j}-D_{j} \partial_{x}^{2} u_{j}+B_{j}\left(u_{j}, \partial_{x} u_{j}\right)+F_{j}\left(u_{j}, u_{j}, u_{j}\right)\right) & =C_{j} \partial_{y}^{2} u_{j},
\end{aligned}
$$

where the constant $v_{j}$ is the speed of the wave in the direction $k$ (the $x$-direction). He also proves the validation of this approximation for a time of the order of $\frac{T_{0}}{\epsilon^{2}}$.

\section{Validity of the KZK-approximation for non-viscous thermo-elastic} media

We now show in what way the KZK equation provides asymptotic solutions of the Navier-Stokes system (1.2), firstly, with $\nu=0$.

We recall that for the case of null viscosity $(\nu=0)$ theorems of existence and stability for the Euler system and for the KZK equation are valid for positive and negative but finite time. Moreover, we note that $z=\epsilon x_{1}$, and $z$ becomes the time variable according to the KZK derivation.

Thus, we first consider the Euler system for $\rho_{\epsilon}\left(x_{1}, x^{\prime}, t\right)$ and $u_{\epsilon}\left(x_{1}, x^{\prime}, t\right)$,

$$
\partial_{t} \rho_{\epsilon}+\operatorname{div}\left(\rho_{\epsilon} u_{\epsilon}\right)=0, \quad \rho_{\epsilon}\left[\partial_{t} u_{\epsilon}+\left(u_{\epsilon} . \nabla\right) u_{\epsilon}\right]=-\nabla p\left(\rho_{\epsilon}\right),
$$

and subsequently, a non-trivial solution $I$ of the problem

$$
c \partial_{\tau z}^{2} I-\frac{(\gamma+1)}{4 \rho_{0}} \partial_{\tau}^{2} I^{2}-\frac{c^{2}}{2} \Delta_{y} I=0
$$

for an initial data

$$
I(t, 0, y)=I_{0}(t, y) \quad\left(\tau=t \text { for } x_{1}=0\right)
$$

$L$-periodic in $t$ with mean value zero. The solution $I$ as a function of $(\tau, z, y)$ is periodic in $\tau$ with period $L$ and mean value zero. We construct in this case for the KZK-approximation a solution for positive and negative $\left(x_{1}, t\right)$, using initial data compact in $x$ for $t=0$.

Theorem 2.1 ensures for initial data $I(t, 0, y) \in H^{s^{\prime}}$ with $s^{\prime}>\left[\frac{n}{2}\right]+1$ the existence of a unique solution $I(\tau, z, y) \in C\left(|z|<R ; H^{s^{\prime}}(\tau \times y)\right.$ ) (for null viscosity $\nu=0$ ), where $R$ corresponds to the limit for the "time existence" interval, i.e., $R \geq 1 / C\left(s^{\prime}, L\right)\left\|I_{0}\right\|_{H^{s^{\prime}}}$. The existence of the smooth solution $U_{\epsilon}=\left(\rho_{\epsilon}, u_{\epsilon}\right)\left(t, x_{1}, x^{\prime}\right)(0 \leq t \leq T)$ of the Euler system (4.1) is due to Theorem 5.1.1 from [6, p. 62].

With the notations

$$
\rho_{\epsilon}=\rho_{0}+\epsilon \tilde{\rho}_{\epsilon}, \quad u_{\epsilon}=\epsilon \tilde{u}_{\epsilon},
$$

we take

$$
p=p\left(\rho_{\epsilon}\right)=c^{2} \epsilon \tilde{\rho}_{\epsilon}+\frac{(\gamma-1) c^{2}}{2 \rho_{0}} \epsilon^{2} \tilde{\rho}_{\epsilon}^{2} .
$$

Then, according to these formulas, the functions we construct are

$$
\begin{aligned}
& v(\tau, z, y)=\frac{c}{\rho_{0}} I(\tau, z, y), \\
& w(\tau, z, y)=-\frac{c^{2}}{\rho_{0}}\left(\int_{0}^{\tau} \nabla_{y} I(s, z, y) d s+\int_{0}^{L} \frac{s}{L} \nabla_{y} I(s, z, y) d s\right),
\end{aligned}
$$




$$
\begin{aligned}
v_{1}(\tau, z, y)= & -\frac{c^{2}}{\rho_{0}}\left(\int_{0}^{\tau} \partial_{z} I(s, z, y) d s+\int_{0}^{L} \frac{s}{L} \partial_{z} I(s, z, y) d s\right) \\
& +\frac{(\gamma-1)}{2 \rho_{0}^{2}} c I^{2}(\tau, z, y)-\frac{c(\gamma-1)}{2 L \rho_{0}^{2}} \int_{0}^{L} I^{2}(\tau, z, y) d \tau
\end{aligned}
$$

In the above formulas the terms containing $\int_{0}^{L}$ correspond to the definition of the operator $\partial_{\tau}^{-1}$ (see equation $(2.3)$ ), which implies that all these functions are $L$-periodic in $\tau$ and have mean value zero.

Next, we introduce the density $\bar{\rho}_{\epsilon}$ and velocity $\bar{u}_{\epsilon}=\left(\bar{u}_{\epsilon, 1}, \bar{u}_{\epsilon}^{\prime}\right)$

$$
\begin{aligned}
& \bar{\rho}_{\epsilon}=\rho_{0}+\epsilon I\left(t-\frac{x_{1}}{c}, \epsilon x_{1}, \sqrt{\epsilon} x^{\prime}\right), \\
& \bar{u}_{\epsilon, 1}=\epsilon\left(v+\epsilon v_{1}\right)\left(t-\frac{x_{1}}{c}, \epsilon x_{1}, \sqrt{\epsilon} x^{\prime}\right), \\
& \bar{u}_{\epsilon}^{\prime}=\epsilon^{\frac{3}{2}} \vec{w}\left(t-\frac{x_{1}}{c}, \epsilon x_{1}, \sqrt{\epsilon} x^{\prime}\right),
\end{aligned}
$$

and eventually the expression:

$$
\bar{U}_{\epsilon}=\left(\bar{\rho}_{\epsilon}, \bar{u}_{\epsilon}\right)=\left(\rho_{0}+\epsilon I, \epsilon\left(v+\epsilon v_{1}, \sqrt{\epsilon} \vec{w}\right)\right)\left(t-\frac{x_{1}}{c}, \epsilon x_{1}, \sqrt{\epsilon} x^{\prime}\right) .
$$

We consider the approximation problem between two systems: the exact system (4.1) and the approximate system obtained from a smooth solution of the KZK equation $(4.2)$,

$$
\begin{aligned}
& \partial_{t} \bar{\rho}_{\epsilon}+\nabla_{x} \cdot\left(\bar{\rho}_{\epsilon} \bar{u}_{\epsilon}\right) \\
= & \epsilon^{3}\left(\rho_{0} \partial_{z} v_{1}+\partial_{z}(I v)-\frac{1}{c} \partial_{\tau}\left(I v_{1}\right)+\nabla_{y}(I w)\right)+\epsilon^{4} \partial_{z}\left(I v_{1}\right), \\
& \bar{\rho}_{\epsilon}\left(\partial_{t} \bar{u}_{\epsilon, 1}+\bar{u}_{\epsilon} \cdot \nabla \cdot \bar{u}_{\epsilon, 1}\right)+\partial_{x_{1}} p\left(\bar{\rho}_{\epsilon}\right) \\
= & \epsilon^{3}\left(I \partial_{\tau} v_{1}-\frac{1}{2 c} I \partial_{\tau} v^{2}+\frac{\rho_{0}}{2} \partial_{z} v^{2}-\frac{\rho_{0}}{c} \partial_{\tau}\left(v v_{1}\right)+\rho_{0} w \nabla_{y} v+\frac{(\gamma-1)}{2 \rho_{0}} c^{2} \partial_{z} I^{2}\right) \\
+ & \epsilon^{4}\left(\frac{I}{2} \partial_{z} v^{2}-\frac{1}{c} I \partial_{\tau}\left(v v_{1}\right)+I w \nabla_{y} v+\rho_{0} \partial_{z}\left(v v_{1}\right)-\frac{\rho_{0}}{2 c} \partial_{\tau} v_{1}^{2}+\rho_{0} w \nabla_{y} v_{1}\right) \\
+ & \left(I \partial_{z}\left(v v_{1}\right)-\frac{1}{2 c} I \partial_{\tau} v_{1}^{2}+I w \nabla_{y} v_{1}+\frac{\rho_{0}}{2} \partial_{z} v_{1}^{2}\right)+\epsilon^{6} I v_{1} \partial_{z} v_{1}, \\
& \bar{\rho}_{\epsilon}\left(\partial_{t} \bar{u}_{\epsilon}^{\prime}+\bar{u}_{\epsilon} \cdot \nabla \cdot \bar{u}_{\epsilon}^{\prime}\right)+\partial_{x^{\prime}} p\left(\bar{\rho}_{\epsilon}\right)=\epsilon^{\frac{5}{2}}\left(\frac{\gamma-1}{2 \rho_{0}} c^{2} \nabla_{y} I^{2}\right) \\
& +\epsilon^{\frac{7}{2}}\left(\rho_{0} v \partial_{z} w-\frac{\rho_{0}}{c} v_{1} \partial_{\tau} w+\frac{\rho_{0}}{2} \nabla_{y} w^{2}-\frac{I}{c} v \partial_{\tau} w\right) \\
& +\epsilon^{\frac{9}{2}}\left(\rho_{0} v_{1} \partial_{z} w+I v \partial_{z} w-\frac{I}{c} v_{1} \partial_{\tau} w+\frac{I}{2} \nabla_{y} w^{2}\right)+\epsilon^{\frac{11}{2}} I v_{1} \partial_{z} w .
\end{aligned}
$$

System (4.12)-(4.14) can be written in the form

$$
\begin{aligned}
& \partial_{t} \bar{\rho}_{\epsilon}+\nabla \cdot\left(\overline{\rho u}_{\epsilon}\right)=R_{1}, \\
& \bar{\rho}_{\epsilon}\left(\partial_{t} \bar{u}_{\epsilon}+\bar{u}_{\epsilon} \cdot \nabla \cdot \bar{u}_{\epsilon}\right)+\nabla p\left(\bar{\rho}_{\epsilon}\right)=\vec{R}_{2},
\end{aligned}
$$


with notation $R_{1}$ for the remaining term of equation (4.12), and $\vec{R}_{2}$ for the remaining term of equations (4.13) and (4.14). To ensure that $R_{1} \in L_{\infty}\left((-R, R) ; L_{2}\right)$ and $\vec{R}_{2}$ is in $\left[L_{\infty}\left((-R, R) ; L_{2}\right)\right]^{2}$, we require that $\partial_{z}^{2} I \in L_{\infty}\left((-R, R) ; L_{2}\right)$ and choose $s>\max \left\{4,\left[\frac{n}{2}\right]+1\right\}$ in Theorem 2.1 .

The existence of the smooth "true" solution of the Euler system (4.1)

$$
U_{\epsilon}=\left(\rho_{\epsilon}, u_{\epsilon}\right)\left(t, x_{1}, x^{\prime}\right) \quad\left(0 \leq t<T_{0}\right) \quad \text { with } \quad \nabla \cdot U_{\epsilon}(\cdot, t) \in C^{0}\left(\left[0, T_{0}\right) ; H^{s-5}\right)
$$

for $s-5>\frac{n}{2}$, with the same initial data $\left.U_{\epsilon}\right|_{t=0}=\left.\bar{U}_{\epsilon}\right|_{t=0}$ is still due to Theorem 5.1.1 from [6, p.62]. Indeed, let us show that the assumptions of this theorem are satisfied.

System (4.1) can be written in the form

$$
\begin{aligned}
& \partial_{t} \rho+\sum_{j=1}^{n} \partial_{x_{j}}\left(\rho u_{j}\right)=0, \\
& \partial_{t}\left(\rho u_{i}\right)+\sum_{j=1}^{n} \partial_{x_{j}}\left(\rho u_{i} u_{j}\right)+\partial_{x_{i}} p(\rho)=0, \quad i=1, \ldots, n,
\end{aligned}
$$

or equivalently for $\widetilde{U}_{\epsilon}=\left(\rho_{\epsilon}, \rho_{\epsilon} u_{\epsilon}\right)$ and $F\left(\widetilde{U}_{\epsilon}\right)=\left(\rho_{\epsilon} u_{\epsilon}, \rho_{\epsilon} u_{\epsilon}^{2}+p\left(\rho_{\epsilon}\right)\right)^{T}$,

$$
\partial_{t} \widetilde{U}_{\epsilon}+\nabla \cdot F\left(\widetilde{U}_{\epsilon}\right)=0
$$

System (4.15) (which is hyperbolic, due to $p^{\prime}(\rho)>0$ ) is called the system of conservation laws. Therefore [6, p.43], by the virtue of $p\left(\rho_{\epsilon}\right)=\rho_{\epsilon}^{2} h^{\prime}\left(\rho_{\epsilon}\right)$, any classical solution of (4.15) satisfies

$$
\partial_{t}\left[\rho_{\epsilon} h\left(\rho_{\epsilon}\right)+\rho_{\epsilon} \frac{|u|^{2}}{2}\right]+\sum_{j=1}^{n} \partial_{x_{j}}\left[\left(\rho_{\epsilon} h\left(\rho_{\epsilon}\right)+\rho_{\epsilon} \frac{|u|^{2}}{2}+p\left(\rho_{\epsilon}\right)\right) u_{j}\right]=0
$$

so that the function $\eta=\rho_{\epsilon} h\left(\rho_{\epsilon}\right)+\rho_{\epsilon} \frac{|u|^{2}}{2}$ is the entropy for system (4.1). In addition, the function $\eta\left(\widetilde{U}_{\epsilon}\right)$ is convex and, consequently, $\eta^{\prime \prime}\left(\widetilde{U}_{\epsilon}\right)$ is positive definite. We also notice from (4.8)-(4.10) and Theorem 2.1 that $v_{1} \in C^{1}\left([-R, R] ; H^{s-4}\right)$, $w \in C^{1}\left([-R, R] ; H^{s-3}\right)$ and $v \in C^{1}\left([-R, R] ; H^{s-2}\right)$ for $s>\left[\frac{n}{2}\right]+1$. Thus for $s-4>$ $\left[\frac{n}{2}\right]+1$ the initial data

$$
\left.\widetilde{U}_{\epsilon}\right|_{t=0}=\left.\bar{U}_{\epsilon}\right|_{t=0}=\widetilde{U}_{0}(x)
$$

are continuously differentiable and bounded on $\left\{\left|x_{1}\right|<\frac{R}{\epsilon}\right\} \times \mathbb{R}^{n-1}$, and $\nabla \widetilde{U}_{0} \in H^{s-5}$ $\left(s-5>\left[\frac{n}{2}\right]\right)$. This shows that we are in the case of Theorem 5.1.1 [6, p.62].

REMARK 4.1. The time $T_{0}$ of the existence of the solution of the Euler system is finite. Thus, the interval $\left[0, T_{0}\right)$ is maximal (see $\left.[6, \mathrm{p} .62]\right)$ in the sense that

$$
\lim _{t \uparrow T_{0}} \sup \|\nabla \cdot U(\cdot, t)\|_{L_{\infty}}=\infty .
$$

Let us determine the order of the blow-up time $T_{0}$. We can observe it with the help of the simplified model of the Euler system presented by Burgers' equation with the initial conditions of the order of $\epsilon$ :

$$
\partial_{t} u+u \partial_{x} u=0,\left.\quad u\right|_{t=0}=\epsilon u_{0} .
$$


It is well known that the first derivative

$$
\nabla_{x} u=\frac{\epsilon \nabla_{x} u_{0}}{1-\epsilon \nabla_{x} u_{0} t}
$$

exists for time

$$
t<\frac{1}{\epsilon\left|\nabla_{x} u_{0}\right|} .
$$

Relating to our problem, we will consider the solution of the Euler equation for the time $t \in\left[0, \frac{T}{\epsilon}\right)$, where $T$ is a constant and $\epsilon$ is small.

Therefore, the solution given by the KZK approximation and a true solution of the Euler system with the same data at time $t=0$ can be compared according to the following theorem.

THEOREM 4.2. Suppose that there exists the solution I of the KZK equation (for an initial data $I_{0}(\tau, 0, y)$ from $\left.H^{s^{\prime}}\right)$ such that

- $I(\tau, z, y)$ is L-periodic and with mean value zero with respect to $\tau$ and defined for $|z| \leq R\left(R \geq 1 / C\left(s^{\prime}, L\right)\left\|I_{0}\right\|_{H^{s^{\prime}}}\right)$ and $y \in \mathbb{R}^{n-1}$,

- for $\Omega=\mathbb{R} / L \mathbb{Z} \times \mathbb{R}_{y}^{n-1}$ and $s^{\prime}>\max \left\{4,\left[\frac{n}{2}\right]+5\right\}$, we assume

$$
z \mapsto I(\tau, z, y) \in C(]-R, R\left[; H^{s^{\prime}}(\Omega)\right) \cap C^{1}(]-R, R\left[; H^{s^{\prime}-2}(\Omega)\right)
$$

(the uniqueness and the existence of such a solution is proved in Theorem 2.1).

Let $\bar{U}_{\epsilon}$ be the approximate solution of the isentropic Euler system deduced from a solution of the KZK equation with the help of equations (4.8)-(4.10) and equations (4.5)-(4.7). Then the function $\bar{U}_{\epsilon}\left(x_{1}, x^{\prime}, t\right)=\bar{U}_{\epsilon}\left(t-\frac{x_{1}}{c}, \epsilon x_{1}, \sqrt{\epsilon} x^{\prime}\right)$ given by formula (4.11) is defined in

$$
\mathbb{R}_{t} \times\left(\Omega_{\epsilon}=\left\{\left|x_{1}\right|<\frac{R}{\epsilon}-c t\right\} \times \mathbb{R}_{x^{\prime}}^{n-1}\right)
$$

and is smooth enough according to the above procedure.

Let us now consider the solution of the Euler system (4.1) in a cone (see Fig. 4.1)

$$
C(t)=\cup_{0<s<t}\{s\} \times Q_{\epsilon}(s)=\left\{x=\left(x_{1}, x^{\prime}\right):\left|x_{1}\right| \leq \frac{R}{\epsilon}-M s, M \geq c, x^{\prime} \in \mathbb{R}^{n-1}\right\}
$$

with the initial data

$$
\left.\left(\bar{\rho}_{\epsilon}-\rho_{\epsilon}\right)\right|_{t=0}=0,\left.\quad\left(\bar{u}_{\epsilon}-u_{\epsilon}\right)\right|_{t=0}=0 .
$$

Consequently, (see [6, p.62]) there exists $T_{0}$ such that for the time interval $0 \leq t \leq \frac{T_{0}}{\epsilon}$ there exists the classical solution $U_{\epsilon}=\left(\rho_{\epsilon}, u_{\epsilon}\right)$ of the Euler system (4.1) in a cone

$$
C(T)=\left\{0<t<T \mid T<\frac{T_{0}}{\epsilon}\right\} \times Q_{\epsilon}(t)
$$

with

$$
\left\|\nabla \cdot U_{\epsilon}\right\|_{L_{\infty}\left(\left[0, \frac{T_{0}}{\epsilon}\left[; H^{\left.s^{\prime}-5\right)}\right.\right.\right.}<\epsilon C \text { for } s^{\prime}>\left[\frac{n}{2}\right]+5 .
$$




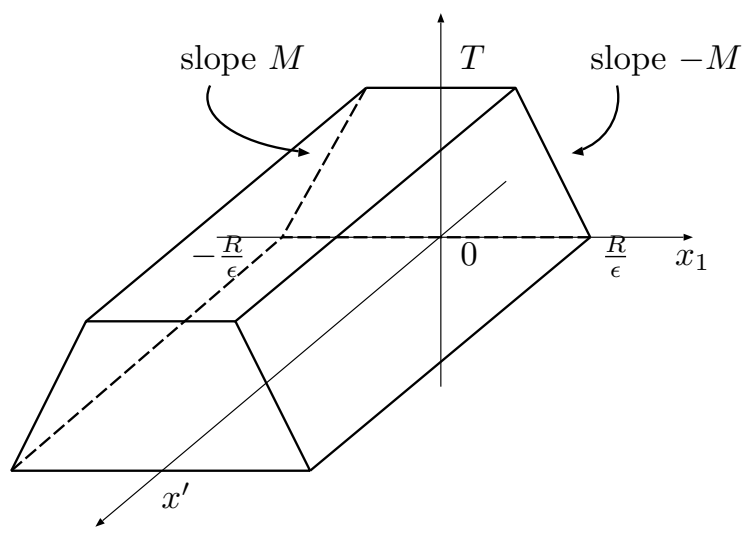

FIG. 4.1. The cone $C(T)$.

Moreover, there exists a constant $C$ such that for any $\epsilon$ small enough, the solutions $\widetilde{U}_{\epsilon} \stackrel{\text { note }}{=}\left(\rho_{\epsilon}, \rho_{\epsilon} u_{\epsilon}\right)$ and $\bar{U}_{\epsilon} \stackrel{\text { note }}{=}\left(\bar{\rho}_{\epsilon}, \bar{\rho}_{\epsilon} \bar{u}_{\epsilon}\right)$, which were determined as above in cone (4.17) with the same initial data (4.16), satisfy the estimate

$$
\left\|\bar{U}_{\epsilon}-\widetilde{U}_{\epsilon}\right\|_{L_{2}\left(Q_{\epsilon}(t)\right)}^{2} \leq \epsilon^{5} e^{C\left\|\nabla \cdot \bar{U}_{\epsilon}\right\|_{L_{\infty}(C(T))}} \leq \epsilon^{5} e^{C \epsilon t} .
$$

REMARK 4.3. Let us introduce the operator $\Lambda=(1-\Delta)^{\frac{1}{2}}$, defined by

$$
\widehat{(\Lambda u)}(\zeta)=\left(1+|\zeta|^{2}\right)^{\frac{1}{2}} \hat{u}(\zeta) .
$$

Consequently, we have

$$
\Lambda^{s}=(1-\Delta)^{\frac{s}{2}}, \quad\|u\|_{H^{s}}=\left\|\Lambda^{s} u\right\|_{L_{2}} .
$$

As soon as both solutions $\bar{U}_{\epsilon}$ and $\widetilde{U}_{\epsilon}$ are in $C\left(\left[0, \frac{T_{0}}{\epsilon}\left[; H^{s^{\prime}-4}\right)\right.\right.$ in the cone for any $s^{\prime}-4>\left[\frac{n}{2}\right]+1$, if in addition $s^{\prime}>8$, we can apply the operator $\Lambda^{\tilde{s}}$ with $\tilde{s}=s^{\prime}-8$ and obtain the same estimate (4.18) but for the norm $\|\cdot\|_{H^{\tilde{s}}\left(Q_{\epsilon}(t)\right)}$.

Proof. Following Dafermos [6, p.43, 61], the convex entropy

$$
\eta\left(\widetilde{U}_{\epsilon}\right)=\rho_{\epsilon} h\left(\rho_{\epsilon}\right)+\rho_{\epsilon} \frac{\left|u_{\epsilon}\right|^{2}}{2} \quad \text { with } \quad h^{\prime}\left(\rho_{\epsilon}\right)=\frac{p\left(\rho_{\epsilon}\right)}{\rho_{\epsilon}^{2}}
$$

is used. With

$$
\widetilde{U}_{\epsilon}=\left(\rho_{\epsilon}, \rho_{\epsilon} u_{\epsilon}\right)^{T}, \quad F\left(\widetilde{U}_{\epsilon}\right)=\left(\rho_{\epsilon} u_{\epsilon}, \rho_{\epsilon} u_{\epsilon}^{2}+p\left(\rho_{\epsilon}\right)\right)^{T} \quad \text { and } \quad q\left(\widetilde{U}_{\epsilon}\right)=u_{\epsilon}\left(\eta\left(\widetilde{U}_{\epsilon}\right)+p\left(\rho_{\epsilon}\right)\right)
$$

we have two systems

$$
\begin{aligned}
& \left\{\begin{array}{l}
\partial_{t} \eta\left(\widetilde{U}_{\epsilon}\right)+\nabla \cdot q\left(\widetilde{U}_{\epsilon}\right)=0, \\
\partial_{t} \widetilde{U}_{\epsilon}+\nabla \cdot F\left(\widetilde{U}_{\epsilon}\right)=0,
\end{array}\right. \\
& \left\{\begin{array}{l}
\partial_{t} \eta\left(\bar{U}_{\epsilon}\right)+\nabla \cdot q\left(\bar{U}_{\epsilon}\right)=\frac{\eta\left(\bar{U}_{\epsilon}\right)+p\left(\bar{\rho}_{\epsilon}\right)}{\bar{\rho}_{\epsilon}} R_{1}+\bar{u}_{\epsilon} \vec{R}_{2}, \\
\partial_{t} \bar{U}_{\epsilon}+\nabla \cdot F\left(\bar{U}_{\epsilon}\right)=\vec{R}
\end{array}\right.
\end{aligned}
$$


where $R_{1}=O\left(\epsilon^{3}\right)$ is the remaining term of the first equation of the Euler system, $\vec{R}_{2}=\left(O\left(\epsilon^{3}\right), O\left(\epsilon^{\frac{5}{2}}\right)\right)=O\left(\epsilon^{\frac{5}{2}}\right)$ is the remaining term of the second equation of the Euler system in two directions, $x_{1}$ and $x^{\prime}$, and $\vec{R}=\left(R_{1}, \overrightarrow{R_{2}}\right)=O\left(\epsilon^{\frac{5}{2}}\right)$. Thus, we have

$$
\frac{\eta\left(\bar{U}_{\epsilon}\right)+p\left(\bar{\rho}_{\epsilon}\right)}{\bar{\rho}_{\epsilon}} R_{1}+\bar{u}_{\epsilon} \vec{R}_{2}=O\left(\epsilon^{3}\right) .
$$

Let us compute

$$
\begin{aligned}
& \partial_{t}\left(\eta\left(\widetilde{U}_{\epsilon}\right)-\eta\left(\bar{U}_{\epsilon}\right)-\eta^{\prime}\left(\bar{U}_{\epsilon}\right)\left(\widetilde{U}_{\epsilon}-\bar{U}_{\epsilon}\right)\right)=-\nabla \cdot\left(q\left(\widetilde{U}_{\epsilon}\right)-q\left(\bar{U}_{\epsilon}\right)\right) \\
& -\vec{R}^{T} \eta^{\prime \prime}(\bar{U})(\widetilde{U}-\bar{U})-\frac{\eta\left(\bar{U}_{\epsilon}\right)+p\left(\bar{\rho}_{\epsilon}\right)}{\bar{\rho}_{\epsilon}} R_{1}-\bar{u}_{\epsilon} \vec{R}_{2} \\
& +\eta^{\prime}\left(\bar{U}_{\epsilon}\right) \nabla \cdot\left(F\left(\widetilde{U}_{\epsilon}\right)-F\left(\bar{U}_{\epsilon}\right)\right)-\eta^{\prime}\left(\bar{U}_{\epsilon}\right) \vec{R}-\left(\partial_{t} \bar{U}_{\epsilon}\right)^{T} \eta^{\prime \prime}\left(\bar{U}_{\epsilon}\right)\left(\widetilde{U}_{\epsilon}-\bar{U}_{\epsilon}\right),
\end{aligned}
$$

and, using the property for the convex entropy $\eta^{\prime \prime}(U) F^{\prime}(U)=\left(F^{\prime}(U)\right)^{T} \eta^{\prime \prime}(U)$, the last term is

$$
\begin{aligned}
& -\left(\partial_{t} \bar{U}_{\epsilon}\right)^{T} \eta^{\prime \prime}\left(\bar{U}_{\epsilon}\right)\left(\widetilde{U}_{\epsilon}-\bar{U}_{\epsilon}\right)=\nabla \cdot \bar{U}_{\epsilon}^{T}\left(F^{\prime}\left(\bar{U}_{\epsilon}\right)\right)^{T} \eta^{\prime \prime}\left(\bar{U}_{\epsilon}\right)\left(\widetilde{U}_{\epsilon}-\bar{U}_{\epsilon}\right) \\
= & \nabla \cdot \bar{U}_{\epsilon}^{T} \eta^{\prime \prime}\left(\bar{U}_{\epsilon}\right) F^{\prime}\left(\bar{U}_{\epsilon}\right)\left(\widetilde{U}_{\epsilon}-\bar{U}_{\epsilon}\right) .
\end{aligned}
$$

We integrate equation (4.20) over the cone $C(t)$ (cf. (4.17)). The use of Green's formula gives

$$
\begin{aligned}
& \int_{\left|x_{1}\right|<\frac{R}{\epsilon}-M t} \int_{\mathbb{R}^{n-1}}\left[\eta\left(\widetilde{U}_{\epsilon}\right)-\eta\left(\bar{U}_{\epsilon}\right)-\eta^{\prime}\left(\bar{U}_{\epsilon}\right)\left(\widetilde{U}_{\epsilon}-\bar{U}_{\epsilon}\right)\right](x, t) d x \\
& -\int_{\left|x_{1}\right|<\frac{R}{\epsilon}} \int_{\mathbb{R}^{n-1}}\left[\eta\left(\widetilde{U}_{\epsilon}\right)-\eta\left(\bar{U}_{\epsilon}\right)-\eta^{\prime}\left(\bar{U}_{\epsilon}\right)\left(\widetilde{U}_{\epsilon}-\bar{U}_{\epsilon}\right)\right](x, 0) d x \\
= & -\int_{\partial C(t)} n_{t}\left[\eta\left(\widetilde{U}_{\epsilon}\right)-\eta\left(\bar{U}_{\epsilon}\right)-\eta^{\prime}\left(\bar{U}_{\epsilon}\right)\left(\widetilde{U}_{\epsilon}-\bar{U}_{\epsilon}\right)\right] d \sigma \\
& -\int_{\partial C(t)} n_{x_{1}}\left[q\left(\widetilde{U}_{\epsilon}\right)-q\left(\bar{U}_{\epsilon}\right)-\eta^{\prime}\left(\bar{U}_{\epsilon}\right)\left(F\left(\widetilde{U}_{\epsilon}\right)-F\left(\bar{U}_{\epsilon}\right)\right)\right] d \sigma \\
& -\int_{C(t)} \nabla \cdot \bar{U}_{\epsilon}^{T} \eta^{\prime \prime}\left(\bar{U}_{\epsilon}\right)\left[F\left(\widetilde{U}_{\epsilon}\right)-F\left(\bar{U}_{\epsilon}\right)-F^{\prime}\left(\bar{U}_{\epsilon}\right)\left(\widetilde{U}_{\epsilon}-\bar{U}_{\epsilon}\right)\right] d x d s \\
& -\int_{C(t)}\left[\frac{\eta\left(\bar{U}_{\epsilon}\right)+p\left(\bar{\rho}_{\epsilon}\right)}{\bar{\rho}_{\epsilon}} R_{1}+\bar{u}_{\epsilon} \vec{R}_{2}+\eta^{\prime}\left(\bar{U}_{\epsilon}\right) \vec{R}-\vec{R}^{T} \eta^{\prime \prime}\left(\bar{U}_{\epsilon}\right)\left(\widetilde{U}_{\epsilon}-\bar{U}_{\epsilon}\right)\right] d x d s .
\end{aligned}
$$

With the help of the facts that the entropy $\eta$ is convex

$$
\eta\left(\widetilde{U}_{\epsilon}\right)-\eta\left(\bar{U}_{\epsilon}\right)-\eta^{\prime}\left(\bar{U}_{\epsilon}\right)\left(\widetilde{U}_{\epsilon}-\bar{U}_{\epsilon}\right) \geq \alpha\left|\widetilde{U}_{\epsilon}-\bar{U}_{\epsilon}\right|^{2},
$$

and the associated entropy flux $q$ is related with $\eta$ by the relation [6, p. 52]

$$
q^{\prime}(U)=\eta^{\prime}(U) F^{\prime}(U)
$$

we obtain that

$$
\begin{aligned}
& q\left(\widetilde{U}_{\epsilon}\right)-q\left(\bar{U}_{\epsilon}\right)=q^{\prime}\left(\bar{U}_{\epsilon}\right)\left(\widetilde{U}_{\epsilon}-\bar{U}_{\epsilon}\right)+O\left(\left|\widetilde{U}_{\epsilon}-\bar{U}_{\epsilon}\right|^{2}\right) \\
= & \eta^{\prime}\left(\bar{U}_{\epsilon}\right) F^{\prime}\left(\bar{U}_{\epsilon}\right)\left(\widetilde{U}_{\epsilon}-\bar{U}_{\epsilon}\right)+O\left(\left|\widetilde{U}_{\epsilon}-\bar{U}_{\epsilon}\right|^{2}\right) .
\end{aligned}
$$


Moreover, using the Taylor expansion, we have

$$
\begin{aligned}
& F\left(\widetilde{U}_{\epsilon}\right)-F\left(\bar{U}_{\epsilon}\right)=F^{\prime}\left(\bar{U}_{\epsilon}\right)\left(\widetilde{U}_{\epsilon}-\bar{U}_{\epsilon}\right)+O\left(\left|\widetilde{U}_{\epsilon}-\bar{U}_{\epsilon}\right|^{2}\right) \\
& F\left(\widetilde{U}_{\epsilon}\right)-F\left(\bar{U}_{\epsilon}\right)-F^{\prime}\left(\bar{U}_{\epsilon}\right)\left(\widetilde{U}_{\epsilon}-\bar{U}_{\epsilon}\right) \leq C\left|\widetilde{U}_{\epsilon}-\bar{U}_{\epsilon}\right|^{2} \\
& q\left(\widetilde{U}_{\epsilon}\right)-q\left(\bar{U}_{\epsilon}\right)-\eta^{\prime}\left(\bar{U}_{\epsilon}\right)\left(F\left(\widetilde{U}_{\epsilon}\right)-F\left(\bar{U}_{\epsilon}\right)\right) \leq C\left|\widetilde{U}_{\epsilon}-\bar{U}_{\epsilon}\right|^{2}
\end{aligned}
$$

We can always choose our cone with the help of a constant $M \geq c$ such that $\alpha n_{t}+$ $C n_{x_{1}}>0$ and

$$
-\int_{\partial C(t)}\left(\alpha n_{t}+C n_{x}\right)\left\|\widetilde{U}_{\epsilon}-\bar{U}_{\epsilon}\right\|_{L_{2}\left(Q_{\epsilon}(s)\right)}^{2} d \sigma<0
$$

Taking the same initial data $\left.\bar{U}_{\epsilon}\right|_{t=0}=\left.\widetilde{U}_{\epsilon}\right|_{t=0}$, we obtain

$$
\left\|\widetilde{U}_{\epsilon}-\bar{U}_{\epsilon}\right\|_{L_{2}\left(Q_{\epsilon}(t)\right)}^{2} \leq C\left\|\nabla \cdot \bar{U}_{\epsilon}\right\|_{L_{\infty}(C(T))} \int_{0}^{t}\left\|\widetilde{U}_{\epsilon}-\bar{U}_{\epsilon}\right\|_{L_{2}\left(Q_{\epsilon}(s)\right)}^{2} d s+K t \epsilon^{5} .
$$

Here the constants $K$ and $C$ do not depend on $t$.

Therefore, applying the Gronwall lemma we have

$$
\left\|\widetilde{U}_{\epsilon}-\bar{U}_{\epsilon}\right\|_{L_{2}\left(Q_{\epsilon}(t)\right)}^{2} \leq K \epsilon^{5} \int_{0}^{t} e^{C(t-s)\left\|\nabla \cdot \bar{U}_{\epsilon}\right\|_{L_{\infty}(C(T))}} d s .
$$

We notice that the difference of the solutions $\widetilde{U}_{\epsilon}-\bar{U}_{\epsilon}$ is of the order of $O(\epsilon)$, moreover, $\nabla \cdot \bar{U}_{\epsilon}=O(\epsilon)$, and the left-hand side of estimation (4.22) is of the order of $O\left(\epsilon^{2}\right)$. Consequently, we have inside of the cone $C(T)$ that

$$
\left\|\widetilde{U}_{\epsilon}-\bar{U}_{\epsilon}\right\|_{L_{2}\left(Q_{\epsilon}(t)\right)}^{2} \leq \epsilon^{5} \int_{0}^{t} e^{C \epsilon(t-s)} d s<\epsilon^{2} \quad \text { for all } t \in[0, T] .
$$

5. Validity of the KZK-approximation for viscous thermo-elastic media

From Theorem 2.1 we see that the solution $u(x, y, t)$ of the KZK equation with the term of viscosity $\beta>0$ or $\nu>0$ is defined globally in time $t>0$ for small enough initial data. The solution $I(\tau, z, y)$ of the KZK equation is the asymptotic form of the Navier-Stokes system. We note that $I(\tau, z, y)=I\left(t-\frac{x_{1}}{c}, \epsilon x_{1}, \sqrt{\epsilon} x^{\prime}\right)$ is defined for $x_{1}>0$ (as $z$ becomes the time variable according to the KZK derivation from section 3 ). Therefore, the approximate domain of validity of the KZK approximation for viscous thermo-elastic media is the half space

$$
\left\{x_{1}>0, \quad t>0, \quad x^{\prime} \in R^{n-1}\right\} .
$$

5.1. Approximation result for the linearized KZK equation. From the Navier-Stokes system

$$
\partial_{t} \hat{\rho}+\operatorname{div}(\hat{\rho} \hat{u})=0, \quad \hat{\rho}\left(\partial_{t} \hat{u}+(\hat{u} \cdot \nabla) \hat{u}\right)=-\nabla \hat{p}(\hat{\rho})+\epsilon \nu \Delta \hat{u},
$$

which is well posed for small enough initial data in the half space (5.1), the isentropic linear Navier-Stokes system can be obtained with the help of the choice

$$
\hat{\rho}=\rho_{0}+\epsilon^{2} \rho, \quad \hat{u}=\epsilon^{2} u, \quad \hat{p}=\hat{p}(\hat{\rho})=\epsilon^{2} p(\rho)=\epsilon^{2} c^{2} \rho,
$$


not taking into account the terms of the order of $O\left(\epsilon^{3}\right)$,

$$
\begin{aligned}
\partial_{t} \rho+\rho_{0} \nabla \cdot u & =0, \\
\rho_{0} \partial_{t} u+\nabla p(\rho) & =\epsilon \nu \Delta u .
\end{aligned}
$$

System (5.3)-(5.4) has a unique global solution.

Combining $\partial_{t}$ and $\nabla$ (or $\nabla \cdot$ ) applied to equations (5.3) and (5.4), we obtain two decoupled linear equations

$$
\begin{aligned}
\partial_{t}^{2} \rho-c^{2} \triangle \rho & =\epsilon \frac{\nu}{\rho_{0}} \triangle \partial_{t} \rho, \\
\partial_{t}^{2} u-c^{2} \nabla \operatorname{div} u & =\epsilon \frac{\nu}{\rho_{0}} \triangle \partial_{t} u .
\end{aligned}
$$

The existence of the smooth solution $u$ of equation (5.6) is proven in the following lemma.

Lemma 5.1. Equation (5.6) has a unique regular global solution in time in the half space $\left\{x_{1}>0, t>0\right\}\left(u \in C^{1}\left([0,+\infty), H^{s-2}\right) \cap C^{2}\left([0,+\infty), H^{s-4}\right), s-1>\left[\frac{n}{2}\right]+1\right)$, with regular initial values

$$
\begin{aligned}
& \left.u\right|_{t=0}=u_{0}=\left(\frac{c}{\rho_{0}} I, \sqrt{\epsilon} \vec{w}\right)\left(-\frac{x_{1}}{c}, \epsilon x_{1}, \sqrt{\epsilon} x^{\prime}\right) \in H^{s-1}, \\
& \left.u_{t}\right|_{t=0}=u_{1}=\left(\frac{c}{\rho_{0}} \partial_{\tau} I, \sqrt{\epsilon} \partial_{\tau} \vec{w}\right)\left(-\frac{x_{1}}{c}, \epsilon x_{1}, \sqrt{\epsilon} x^{\prime}\right) \in H^{s-2},
\end{aligned}
$$

and boundary conditions

$$
\left.u\right|_{x_{1}=0}=u_{b}=\left(\frac{c}{\rho_{0}} I, \sqrt{\epsilon} \vec{w}\right)\left(t, 0, \sqrt{\epsilon} x^{\prime}\right) \in H^{s-1}
$$

(in addition $u_{b}$ is L-periodic and with mean value zero to respect to $t$ ).

Proof. If $u_{b}=0$, the relation

$$
\begin{aligned}
& \frac{1}{2} \frac{d}{d t}\left(\int_{x_{1}>0}\left|\partial_{t} u\right|^{2} d x+c^{2} \int_{x_{1}>0}|\nabla \cdot u|^{2} d x\right)+\frac{\epsilon \nu}{\rho_{0}} \int_{x_{1}>0}\left|\nabla \cdot \partial_{t} u\right|^{2} d s \\
& -c^{2} \int_{x_{1}=0} \nabla \cdot u \partial_{t} u d x^{\prime}-\frac{\epsilon \nu}{\rho_{0}} \int_{x_{1}=0} \nabla \cdot \partial_{t} u \partial_{t} u d x^{\prime}=0,
\end{aligned}
$$

gives

$$
\frac{d}{d t} \int_{x_{1}>0}\left(\left|\partial_{t} u\right|^{2}+c^{2}|\nabla \cdot u|^{2}\right) d x \leq 0 .
$$

If we take $u_{b} \neq 0$, then we can write $u=v+\varphi$, such that $\left.v\right|_{t=0},\left.v_{t}\right|_{t=0}$ are the same as for $u$ and equal to zero at the boundary and $\varphi$ is a function with a compact support with null initial conditions and the same boundary condition as $u$. We can construct such $\varphi$ using the Trace Theorem, due to the regularity of $u_{b} \in H^{s-1}, s-1>\left[\frac{n}{2}\right]+1$.

Let us put $u=v+\varphi$ in equation (5.6)

$$
\partial_{t}^{2}(v+\varphi)-c^{2} \nabla \operatorname{div}(v+\varphi)=\epsilon \frac{\nu}{\rho_{0}} \triangle \partial_{t}(v+\varphi) .
$$

From which we obtain

$$
\partial_{t}^{2} v-c^{2} \nabla \operatorname{div} v-\epsilon \frac{\nu}{\rho_{0}} \triangle \partial_{t} v=g(\varphi) .
$$


We now multiply equation (5.7) by $\partial_{t} v$ and integrate over $x$ :

$$
\begin{aligned}
& \frac{1}{2} \frac{d}{d t}\left(\int_{x_{1}>0}\left|\partial_{t} v\right|^{2} d x+c^{2} \int_{x_{1}>0}|\nabla \cdot v|^{2} d x\right)+\frac{\epsilon \nu}{\rho_{0}} \int_{x_{1}>0}\left|\nabla \cdot \partial_{t} v\right|^{2} d s \\
& \leq \frac{1}{2} \int_{x_{1}>0}|g(\varphi)|^{2} d x+\frac{1}{2} \int_{x_{1}>0}\left|\partial_{t} v\right|^{2} d x .
\end{aligned}
$$

Applying the Gronwall Lemma for the equality type $y^{\prime} \leq a+y$, we obtain the global existence in time of the solution of equation (5.6). Therefore, by a standard approach, we obtain the regularity of the solution.

REMARK 5.2. Note that, for $u=\left(u_{1}, u^{\prime}\right)$, the first component of the velocity $u_{1}$ is more regular than $u^{\prime}: u_{1} \in C^{1}\left([0,+\infty), H^{s-1}\right) \cap C^{2}\left([0,+\infty), H^{s-3}\right)$, which is an unneeded property in the following.

Having the solution $u$ of equation (5.6) with the boundary condition $\left.u\right|_{x_{1}=0}=u_{b}$, we can find $\rho$ from equation (5.3) according to the formula

$$
\rho(x, t)=\rho(x, 0)-\rho_{0} \int_{0}^{t} \nabla \cdot u(x, s) d s .
$$

This $\rho$ from equation (5.8) satisfies equation (5.5) with the boundary condition

$$
\left.\partial_{t} \rho\right|_{x_{1}=0}=-\left.\rho_{0} \nabla \cdot u\right|_{x_{1}=0} .
$$

Thus, we can consider the following system instead of system (5.3)-(5.4),

$$
\begin{aligned}
\partial_{t} \rho+\rho_{0} \nabla \cdot u & =0, \\
\partial_{t}^{2} u-c^{2} \nabla \operatorname{div} u & =\epsilon \frac{\nu}{\rho_{0}} \triangle \partial_{t} u .
\end{aligned}
$$

If we change equation (5.5) to the variables $(\tau, z, y)$, we obtain the linear part of the KZK equation for $\bar{\rho}\left(t, x_{1}, x^{\prime}\right)=I\left(t-\frac{x_{1}}{c}, \epsilon x_{1}, \sqrt{\epsilon} x^{\prime}\right)$

$$
\begin{aligned}
& \partial_{t}^{2} \bar{\rho}-c^{2} \triangle \bar{\rho}-\epsilon \frac{\nu}{\rho_{0}} \triangle \partial_{t} \bar{\rho}=\epsilon\left(2 c \partial_{\tau z}^{2} I-c^{2} \triangle_{y} I-\frac{\nu}{\rho_{0} c^{2}} \partial_{\tau}^{3} I\right) \\
& +\epsilon^{2}\left(-c^{2} \partial_{z}^{2} I+2 \frac{\nu}{\rho_{0} c} \partial_{\tau}^{2} \partial_{z} I-\frac{\nu}{\rho_{0}} \triangle_{y} \partial_{\tau} I\right)-\epsilon^{3} \frac{\nu}{\rho_{0}} \partial_{z}^{2} \partial_{\tau} I
\end{aligned}
$$

Suppose that $I$ is the smooth periodic solution of the linear KZK equation

$$
2 c \partial_{\tau z}^{2} I-c^{2} \triangle_{y} I-\frac{\nu}{\rho_{0} c^{2}} \partial_{\tau}^{3} I=0,
$$

then we have

$$
\partial_{t}^{2} \bar{\rho}-c^{2} \triangle \bar{\rho}-\epsilon \frac{\nu}{\rho_{0}} \triangle \partial_{t} \bar{\rho}=\epsilon^{2}\left(-c^{2} \partial_{z}^{2} I+2 \frac{\nu}{\rho_{0} c} \partial_{\tau}^{2} \partial_{z} I-\frac{\nu}{\rho_{0}} \triangle_{y} \partial_{\tau} I\right)-\epsilon^{3} \frac{\nu}{\rho_{0}} \partial_{z}^{2} \partial_{\tau} I .
$$

An equation of the same form holds for $\bar{u}=(v, \sqrt{\epsilon} \vec{w})$

$$
\partial_{t}^{2} \bar{u}-c^{2} \nabla \operatorname{div} \bar{u}-\epsilon \frac{\nu}{\rho_{0}} \triangle \partial_{t} \bar{u}=\left\{\begin{array}{l}
\epsilon^{2}\left(-c^{2} \partial_{z}^{2} v-c^{2} \nabla_{y} \cdot \partial_{z} \vec{w}+2 \frac{\nu}{\rho_{0} c} \partial_{\tau}^{2} \partial_{z} v-\frac{\nu}{\rho_{0}} \triangle_{y} \partial_{\tau} v\right) \\
-\epsilon^{3} \frac{\nu}{\rho_{0}} \partial_{z}^{2} \partial_{\tau} v \\
-\epsilon^{\frac{3}{2}} c \partial_{\tau z}^{2} \vec{w}+\epsilon^{\frac{5}{2}}\left(2 \frac{\nu}{\rho_{0} c} \partial_{\tau}^{2} \partial_{z} \vec{w}-\frac{\nu}{\rho_{0}} \triangle_{y} \partial_{\tau} \vec{w}\right)-\epsilon^{\frac{7}{2}} \frac{\nu}{\rho_{0}} \partial_{z}^{2} \partial_{\tau} \vec{w}
\end{array}\right.
$$


where the functions $v$ and $\vec{w}$ are constructed according to formulas (4.5) and (4.6). Therefore, for the exact system (5.9)-(5.10), the approximate system has the form

$\partial_{t} \bar{\rho}+\rho_{0} \nabla \cdot \bar{u}=\epsilon \rho_{0}\left(\partial_{z} v+\nabla_{y} \vec{w}\right)$,

$\partial_{t}^{2} \bar{u}-c^{2} \nabla \operatorname{div} \bar{u}-\epsilon \frac{\nu}{\rho_{0}} \triangle \partial_{t} \bar{u}=\left\{\begin{array}{l}\epsilon^{2}\left(-c^{2} \partial_{z}^{2} v-c^{2} \nabla_{y} \cdot \partial_{z} \vec{w}+2 \frac{\nu}{\rho_{0} c} \partial_{\tau}^{2} \partial_{z} v-\frac{\nu}{\rho_{0}} \triangle_{y} \partial_{\tau} v\right) \\ -\epsilon^{3} \frac{\nu}{\rho_{0}} \partial_{z}^{2} \partial_{\tau} v, \\ -\epsilon^{\frac{3}{2}} c \partial_{\tau z}^{2} \vec{w}+\epsilon^{\frac{5}{2}}\left(2 \frac{\nu}{\rho_{0} c} \partial_{\tau}^{2} \partial_{z} \vec{w}-\frac{\nu}{\rho_{0}} \triangle_{y} \partial_{\tau} \vec{w}\right)-\epsilon^{\frac{7}{2}} \frac{\nu}{\rho_{0}} \partial_{z}^{2} \partial_{\tau} \vec{w} .\end{array}\right.$

In this case we easily obtain the following theorem.

THEOREM 5.3. Let $I(\tau, z, y)$ be a solution of the linear KZK Equation (5.11), Lperiodic with mean value zero with respect to $\tau$, and defined in the half space $\{z>0$, $\left.\tau \in \mathbb{R} /(L \mathbb{Z}), y \in \mathbb{R}^{n-1}\right\}$, which decays [24] for $z \rightarrow \infty$.

Assume that for $\Omega=(\mathbb{R} /(L \mathbb{Z})) \times \mathbb{R}_{y}^{n-1}$,

$$
z \mapsto I(\tau, z, y) \in C\left(\left[0, \infty\left[; H^{s^{\prime}}(\Omega)\right) \cap C^{1}\left(\left[0, \infty\left[; H^{s^{\prime}-2}(\Omega)\right),\right.\right.\right.\right.
$$

with $s^{\prime}>\max \left\{6,\left[\frac{n}{2}\right]+2\right\}$.

Let $\bar{U}_{\epsilon}=(\bar{\rho}, \bar{u})$ be the smooth solution of the approximate system (5.14)-(5.15) deduced from a solution of the KZK equation with the help of equations (4.5)-(4.6). Then the function $\bar{U}_{\epsilon}\left(x_{1}, x^{\prime}, t\right)=\bar{U}_{\epsilon}\left(t-\frac{x_{1}}{c}, \epsilon x_{1}, \sqrt{\epsilon} x^{\prime}\right)$, given by the formula

$$
\bar{U}_{\epsilon}\left(x_{1}, x^{\prime}, t\right)=(I,(v, \sqrt{\epsilon} \vec{w}))\left(t-\frac{x_{1}}{c}, \epsilon x_{1}, \sqrt{\epsilon} x^{\prime}\right),
$$

is defined in the half space

$$
\left\{x_{1}>0, x^{\prime} \in \mathbb{R}^{n-1}, t>0\right\}
$$

and is smooth enough according to the above procedure.

If $U=(\rho, u)$ is the solution of system (5.9)-(5.10) in the half space with the same small enough initial data and boundary conditions

$$
\begin{aligned}
& \left.\left(\bar{u}_{\epsilon}-u\right)\right|_{t=0}=\left.\partial_{t}\left(\bar{u}_{\epsilon}-u\right)\right|_{t=0}=0,\left.\quad\left(\bar{\rho}_{\epsilon}-\rho\right)\right|_{t=0}=0, \\
& \left.\left(\overline{u_{\epsilon}}-u\right)\right|_{x_{1}=0}=0,\left.\quad \partial_{t} \rho\right|_{x_{1}=0}=-\left.\rho_{0} \nabla \cdot u\right|_{x_{1}=0},
\end{aligned}
$$

then there exists a constant $C>0$ such that

$$
\int_{x_{1}>0}\left|\partial_{t}\left(\bar{u}_{\epsilon}-u\right)\right|^{2}+\left|\nabla \cdot\left(\bar{u}_{\epsilon}-u\right)\right|^{2} d x_{1} d x^{\prime} \leq \epsilon^{3} C t^{2},
$$

and

$$
\int_{x_{1}>0}\left|\bar{\rho}_{\epsilon}-\rho\right|^{2} d x_{1} d x^{\prime} \leq \epsilon^{2} C t^{2} .
$$

The left-hand sides of estimates (5.17) and (5.18) remain smaller than order $O(1)$ for the time $0<t<\frac{T}{\epsilon^{\frac{3}{2}}}$ and $0<t<\frac{T}{\epsilon}$ respectively.

The approximation result is true for the solution $\widetilde{U}$ of the linearized system (5.3)-(5.4) since $\widetilde{U} \equiv U$ (with $\left.\left.\partial_{t} \rho\right|_{x_{1}=0}=-\left.\rho_{0} \nabla \cdot u\right|_{x_{1}=0}\right)$. 
Proof. For the difference $\bar{u}-u$ we have

$$
\partial_{t}^{2}(\bar{u}-u)-c^{2} \triangle(\bar{u}-u)=\epsilon \frac{\nu}{\rho_{0}} \triangle \partial_{t}(\bar{u}-u)+\epsilon^{\frac{3}{2}} \vec{R},
$$

where

$$
\vec{R}=\left\{\begin{array}{l}
\epsilon^{2}\left(-c^{2} \partial_{z}^{2} v-c^{2} \nabla_{y} \cdot \partial_{z} \vec{w}+2 \frac{\nu}{\rho_{0} c} \partial_{\tau}^{2} \partial_{z} v-\frac{\nu}{\rho_{0}} \triangle_{y} \partial_{\tau} v\right)-\epsilon^{3} \frac{\nu}{\rho_{0}} \partial_{z}^{2} \partial_{\tau} v \\
-\epsilon^{\frac{3}{2}} c \partial_{\tau z}^{2} \vec{w}+\epsilon^{\frac{5}{2}}\left(2 \frac{\nu}{\rho_{0} c} \partial_{\tau}^{2} \partial_{z} \vec{w}-\frac{\nu}{\rho_{0}} \triangle_{y} \partial_{\tau} \vec{w}\right)-\epsilon^{\frac{7}{2}} \frac{\nu}{\rho_{0}} \partial_{z}^{2} \partial_{\tau} \vec{w}
\end{array}\right.
$$

is the remaining term of equation (5.13). $\vec{R}$ is bounded, under the smoothness hypotheses of the KZK solution $I$, at least in $L_{\infty}\left(\left[0, \infty\left[, L_{2}\right)\right.\right.$.

Multiplying equation $(5.19)$ by $\partial_{t}(\bar{u}-u)$, we obtain

$$
\begin{aligned}
& \frac{d}{d t} \int_{x_{1}>0}\left(\left|\partial_{t}(\bar{u}-u)\right|^{2}+c^{2}|\nabla \cdot(\bar{u}-u)|^{2}\right) d x_{1} d x^{\prime}-c^{2} \int_{x_{1}=0} \partial_{t}(\bar{u}-u) \cdot \nabla \cdot(\bar{u}-u) d x^{\prime} \\
= & \epsilon \frac{\nu}{\rho_{0}} \int_{x_{1}=0} \partial_{t}(\bar{u}-u) \nabla \cdot \partial_{t}(\bar{u}-u) d x^{\prime}-\epsilon \frac{\nu}{\rho_{0}} \int_{x_{1}>0}\left|\nabla \cdot \partial_{t}(\bar{u}-u)\right|^{2} d x_{1} d x^{\prime} \\
& +\epsilon^{\frac{3}{2}} \int_{x_{1}>0} \vec{R} \partial_{t}(\bar{u}-u) d x_{1} d x^{\prime} .
\end{aligned}
$$

At the same time we have

$$
\frac{\epsilon \nu}{\rho_{0}} \int_{x_{1}=0} \partial_{t}(\bar{u}-u) \nabla \cdot \partial_{t}(\bar{u}-u) d x^{\prime}+c^{2} \int_{x_{1}=0} \partial_{t}(\bar{u}-u) \cdot \nabla \cdot(\bar{u}-u) d x^{\prime}=0,
$$

because we can choose $\left.\bar{u}\right|_{x_{1}=0}=\left.u\right|_{x_{1}=0}($ cf. (5.16)). Therefore, we find

$$
\begin{aligned}
& \frac{d}{d t} \int_{x_{1}>0}\left(\left|\partial_{t}(\bar{u}-u)\right|^{2}+c^{2}|\nabla \cdot(\bar{u}-u)|^{2}\right) d x_{1} d x^{\prime} \\
\leq & \epsilon^{\frac{3}{2}} C\left(\int_{x_{1}>0}\left|\partial_{t}(\bar{u}-u)\right|^{2}+c^{2}|\nabla \cdot(\bar{u}-u)|^{2} d x_{1} d x^{\prime}\right)^{\frac{1}{2}} .
\end{aligned}
$$

The above estimate has the form

$$
\frac{d}{d t}(\sqrt{E})^{2} \leq C \epsilon^{\frac{3}{2}} \sqrt{E} \Rightarrow \frac{d}{d t} \sqrt{E} \leq C \epsilon^{\frac{3}{2}}
$$

and consequently, we have

$$
\frac{d}{d t}\left(\int_{x_{1}>0}\left|\partial_{t}(\bar{u}-u)\right|^{2}+c^{2}|\nabla \cdot(\bar{u}-u)|^{2} d x_{1} d x^{\prime}\right)^{\frac{1}{2}} \leq C \epsilon^{\frac{3}{2}} .
$$

Relation (5.20) implies estimate (5.17).

In our construction the expression $\sqrt{E}=O(1)$ because $\bar{u}=O(1), u=O(1)$, and $(\bar{u}-u)=O(1)$, which remains true in estimate (5.17) for the time $0<t<\frac{T}{\epsilon^{\frac{3}{2}}}$.

Moreover, for $(\bar{\rho}-\rho)$ we have the relation

$$
\partial_{t}(\bar{\rho}-\rho)+\rho_{0} \nabla \cdot(\bar{u}-u)=\epsilon \rho_{0}\left(\partial_{z} v+\nabla_{y} \vec{w}\right) .
$$

We multiply equation $(5.21)$ by $(\bar{\rho}-\rho)$ in $L_{2}\left(\left\{x_{1}>0\right\}\right)$

$$
\frac{d}{d t} \int_{x_{1}>0}|\bar{\rho}-\rho|^{2} d x+\rho_{0} \int_{x_{1}>0}(\bar{\rho}-\rho) \nabla \cdot(\bar{u}-u) d x=\epsilon \rho_{0} \int_{x_{1}>0} R(\bar{\rho}-\rho) d x,
$$

and using estimate (5.17) for the time $t<\frac{T}{\epsilon^{\frac{3}{2}}}$, we obtain the estimate

$$
\|\bar{\rho}-\rho\|_{L_{2}\left(\left\{x_{1}>0\right\}\right)} \leq \epsilon C t,
$$

which requires $\bar{\rho}-\rho=O(1)$ for the time $t<\frac{T}{\epsilon}$. 
5.2. Entropy estimate for the isentropic Navier-Stokes equation on the half space and the associated existence result. First, we consider the system

$$
\partial_{t} \rho_{\epsilon}+\operatorname{div}\left(\rho_{\epsilon} u_{\epsilon}\right)=0, \quad \rho_{\epsilon}\left[\partial_{t} u_{\epsilon}+\left(u_{\epsilon} \cdot \nabla\right) u_{\epsilon}\right]=-\nabla p\left(\rho_{\epsilon}\right)+\epsilon \nu \Delta u_{\epsilon}
$$

and subsequently, a non trivial solution $I$ of the problem

$$
c \partial_{\tau z}^{2} I-\frac{(\gamma+1)}{4 \rho_{0}} \partial_{\tau}^{2} I^{2}-\frac{\nu}{2 c^{2} \rho_{0}} \partial_{\tau}^{3} I-\frac{c^{2}}{2} \Delta_{y} I=0
$$

for an initial data

$$
I(t, 0, y)=I_{0}(t, y) \quad\left(\tau=t \text { for } x_{1}=0\right)
$$

$L$-periodic in $t$ with mean value zero. The solution $I$ as a function of $(\tau, z, y)$ is periodic in $\tau$ of period $L$ and mean value zero. Theorem 2.1 implies that for any initial data $I_{0}$, defined in $\left(\mathbb{R}_{t} / L \mathbb{Z}\right) \times \Omega_{y}$ with small enough $H^{s}\left(s>\left[\frac{n}{2}\right]+1\right)$ norm (with respect to $\nu$ ), there exists a unique solution of the KZK Equation (5.23), which decays for $z \rightarrow \infty$ (see $[24])$.

We still consider our problem in the half space

$$
\left\{x_{1}>0, \quad t>0, \quad x^{\prime} \in \mathbb{R}^{n-1}\right\} .
$$

We take

$$
\begin{aligned}
v_{1}(\tau, z, y)= & -\frac{c^{3}}{2 \rho_{0}} \partial_{\tau}^{-2} \triangle_{y} I(\tau, z, y)+\frac{(\gamma-3)}{4 \rho_{0}^{2}} c I^{2}(\tau, z, y) \\
& -\frac{c(\gamma-3)}{4 L \rho_{0}^{2}} \int_{0}^{L} I^{2}(\tau, z, y) d \tau+\frac{\nu}{2 c \rho_{0}^{2}} \partial_{\tau} I(\tau, z, y)
\end{aligned}
$$

Next we construct the density and velocities (4.8)-(4.10) and construct the function $\bar{U}$ given by (4.11).

In particular, for $t=0$ we have functions defined for $x_{1}>0$ because $I$ is well-defined for any $z>0$

$$
\begin{aligned}
& \bar{\rho}_{\epsilon}\left(0, x_{1}, x^{\prime}\right)=\rho_{0}+\epsilon I\left(-\frac{x_{1}}{c}, \epsilon x_{1}, \sqrt{\epsilon} x^{\prime}\right), \\
& \bar{u}_{\epsilon}\left(0, x_{1}, x^{\prime}\right)=\left(\bar{u}_{\epsilon, 1}, \bar{u}_{\epsilon}^{\prime}\right)\left(-\frac{x_{1}}{c}, \epsilon x_{1}, \sqrt{\epsilon} x^{\prime}\right)
\end{aligned}
$$

and for $x_{1}=0$ we have $L$-periodic functions with mean value zero

$$
\begin{aligned}
& \bar{\rho}_{\epsilon}\left(t, 0, x^{\prime}\right)=\rho_{0}+\epsilon I\left(t, 0, \sqrt{\epsilon} x^{\prime}\right), \\
& \bar{u}_{\epsilon}\left(t, 0, x^{\prime}\right)=\left(\bar{u}_{\epsilon, 1}, \bar{u}_{\epsilon}^{\prime}\right)\left(t, 0, \sqrt{\epsilon} x^{\prime}\right) .
\end{aligned}
$$

It is important to notice that the solution $u$ of system (5.22) is small on the boundary: $\left.u\right|_{x_{1}=0}=\left.\epsilon \tilde{u}_{\epsilon}\right|_{x_{1}=0}$. Therefore, we have $|u|_{x_{1}=0} \mid<c$, which corresponds to the "subsonic" boundary case. More precisely, when the first velocity component is positive $\left.u_{1}\right|_{x_{1}=0}>0$, we have a subsonic inflow boundary condition, and when it is negative $\left.u_{1}\right|_{x_{1}=0}<0$, we have a subsonic outflow boundary condition.

We also notice that, due to equation (5.25), the first component of the velocity $\bar{u}_{1}$ on the boundary has the following form

$$
\begin{aligned}
\left.\bar{u}_{1}\right|_{x_{1}=0} & =\left(\epsilon \frac{c}{\rho_{0}} I+\epsilon^{2} G(I)\right)\left(t, 0, \sqrt{\epsilon} x^{\prime}\right)=\left.\left(\epsilon \frac{c}{\rho_{0}} I+\epsilon^{2} G(I)\right)\right|_{z=0} \\
& =\epsilon \frac{c}{\rho_{0}} I_{0}(t, y)+\epsilon^{2} G\left(I_{0}\right)(t, y),
\end{aligned}
$$


where

$$
G(I)=-\frac{c^{3}}{2 \rho_{0}} \partial_{\tau}^{-2} \triangle_{y} I+\frac{(\gamma-3)}{4 \rho_{0}^{2}} c I^{2}-\frac{c(\gamma-3)}{4 L \rho_{0}^{2}} \int_{0}^{L} I^{2} d \tau+\frac{\nu}{2 c \rho_{0}^{2}} \partial_{\tau} I .
$$

Therefore, the boundary conditions for $\bar{u}_{1}$ are defined by the initial conditions for the KZK equation, are $L$-periodic in $t$, and have mean value zero. In addition, the sign of $\left.\bar{u}_{1}\right|_{x_{1}=0}$ is the same as the sign of $I_{0}$ (because the term $G\left(I_{0}\right)$ is of a higher order of smallness on $\epsilon$ ).

REMARK 5.4. As the viscosity term is $\epsilon \nu \triangle u$, where $\epsilon$ is fixed small enough parameter, $\nu$ is a constant, and in our case $u$ is of the order of $\epsilon$, the boundary layer phenomenon can be excluded.

TheOREM 5.5. Suppose that the initial data of the KZK Cauchy problem $I_{0}(t, y)=$ $I_{0}\left(t, \sqrt{\epsilon} x^{\prime}\right)$ is such that

1. $I_{0}$ is L-periodic in $t$ with mean value zero,

2. for fixed $t, I_{0}$ has the same sign for all $y \in \mathbb{R}^{n-1}$, and for $\left.t \in\right] 0, L[$ the sign changes, i.e., $I_{0}=0$ a finite number of times,

3. $I_{0}(t, y) \in H^{s^{\prime}}\left(\{t \geq 0\} \times \mathbb{R}^{n-1}\right)$ for $s^{\prime}>\max \left\{6,\left[\frac{n}{2}\right]+1\right\}$,

4. $I_{0}$ is sufficiently small in the sense of Theorem 2.1 such that

$$
\left\|I_{0}\right\|_{H^{s^{\prime}}}<\frac{\nu}{2 c^{2} \rho_{0}} \frac{C_{1}(L)}{C_{2}\left(s^{\prime}\right)} \quad \text { (see [24, p. 20]), }
$$

and $I_{0}=\epsilon^{\alpha} \tilde{I}_{0}, \alpha \geq 0$.

Consequently, there exists a unique global solution in time $I(\tau, z, y)$ of (3.24) for $z=$ $\epsilon x_{1}>0$, moreover, the functions $\bar{\rho}_{\epsilon}, \bar{u}_{\epsilon}=\left(\bar{u}_{\epsilon, 1}, \bar{u}_{\epsilon}^{\prime}\right)$, defined by (4.8)-(4.10) in the half space (5.24) are smooth with $\Omega=(\mathbb{R} /(L \mathbb{Z})) \times \mathbb{R}_{y}^{n-1}$ :

$$
\begin{gathered}
\bar{\rho}_{\epsilon} \in C\left(\left[0, \infty\left[, H^{s^{\prime}}(\Omega)\right) \cap C^{1}\left(\left[0, \infty\left[; H^{s^{\prime}-2}(\Omega)\right),\right.\right.\right.\right. \\
\bar{u}_{\epsilon} \in C\left(\left[0, \infty\left[; H^{s^{\prime}-2}(\Omega)\right) \cap C^{1}\left(\left[0, \infty\left[; H^{s^{\prime}-4}(\Omega)\right) .\right.\right.\right.\right.
\end{gathered}
$$

The Navier-Stokes system (5.22) in the half space with initial data (4.16) and following boundary conditions

$$
\left.\left(\bar{u}_{\epsilon}-u_{\epsilon}\right)\right|_{x_{1}=0}=0,
$$

with positive first component of the velocity $\left.u_{\epsilon, 1}\right|_{x_{1}=0}>0$ (i.e. at points where the fluid enters the domain) has the additional boundary condition

$$
\left.\left(\bar{\rho}_{\epsilon}-\rho_{\epsilon}\right)\right|_{x_{1}=0}=0 .
$$

When $\left.u_{\epsilon, 1}\right|_{x_{1}=0} \leq 0$ there is no any boundary condition for $\rho_{\epsilon}$.

Then, there exists a constant $T_{0}>0$ such that for all $t<\frac{T_{0}}{\epsilon^{2+\alpha}}$ there exists a unique solution $U_{\epsilon}=\left(\rho_{\epsilon}, u_{\epsilon}\right)$ of the Navier-Stokes system (5.22) with the same smoothness as (5.29) and (5.30).

REMARK 5.6. Considering that the boundary conditions for the Navier-Stokes system are periodic and of mean value zero in $t,\left.u_{1}\right|_{x_{1}=0}$ changes the sign and the inflow part of 
the boundary goes after the inflow one and so on. This avoids a change in the type of the boundary condition applied to the tangential variables. Moreover, Zabolotskaya [5] takes as the initial conditions for the KZK equation (which correspond to the boundary condition for $u_{1}$ ) the expression

$$
I(\tau, 0, y)=-F(y) \sin \tau
$$

where $F(y)$ is the amplitude distribution of the beam. For a Gaussian beam [5], this reduces to

$$
F(y)=e^{-y^{2}}
$$

while for a beam with a polynomial amplitude we have

$$
F(y)=\left\{\begin{array}{l}
\left(1-y^{2}\right)^{2}, \quad y \leq 1 \\
0, \quad y>1
\end{array}\right.
$$

Proof. We use the fact that the entropy for the isentropic Euler system $\eta\left(\widetilde{U}_{\epsilon}\right)$, defined by (see equation (4.19))

$$
\eta\left(\widetilde{U}_{\epsilon}\right)=\rho_{\epsilon} h\left(\rho_{\epsilon}\right)+\rho_{\epsilon} \frac{\left|u_{\epsilon}\right|^{2}}{2}=H\left(\rho_{\epsilon}\right)+\frac{1}{\rho_{\epsilon}} \frac{m^{2}}{2} \quad \text { with } \quad h^{\prime}\left(\rho_{\epsilon}\right)=\frac{p\left(\rho_{\epsilon}\right)}{\rho_{\epsilon}^{2}}, \quad u_{\epsilon}=\frac{m}{\rho_{\epsilon}},
$$

is a convex function [6]. The first and second derivatives of $\eta\left(\widetilde{U}_{\epsilon}\right)$ have the forms:

$$
\begin{aligned}
& \eta^{\prime}\left(\widetilde{U}_{\epsilon}\right)=\left[\begin{array}{c}
\left.H^{\prime}\left(\rho_{\epsilon}\right)-\frac{1}{\rho_{\epsilon}^{2}} \frac{m^{2}}{2}\right]^{\frac{m}{\rho_{\epsilon}}}
\end{array}\right]^{T}=\left[\begin{array}{c}
H^{\prime}\left(\rho_{\epsilon}\right)-\frac{u_{\epsilon}^{2}}{2} \\
u_{\epsilon}
\end{array}\right]^{T}, \\
& \eta^{\prime \prime}\left(\widetilde{U}_{\epsilon}\right)=\left[\begin{array}{cc}
H^{\prime \prime}\left(\rho_{\epsilon}\right)+\frac{m^{2}}{\rho_{\epsilon}^{3}}, & -\frac{m}{\rho_{\epsilon}^{2}} \\
-\frac{m}{\rho_{\epsilon}^{2}}, & \frac{1}{\rho_{\epsilon}}
\end{array}\right]=\left[\begin{array}{cc}
H^{\prime \prime}\left(\rho_{\epsilon}\right)+\frac{u_{\epsilon}^{2}}{\rho_{\epsilon}},-\frac{u_{\epsilon}}{\rho_{\epsilon}} \\
-\frac{u_{\epsilon}}{\rho_{\epsilon}}, & \frac{1}{\rho_{\epsilon}}
\end{array}\right] .
\end{aligned}
$$

Assuming $\widetilde{U}_{\epsilon}=\left(\rho_{\epsilon}, \rho_{\epsilon} u_{\epsilon}\right)^{T}=\left(\rho_{\epsilon}, m\right)^{T}$, we can rewrite the Navier-Stokes system (5.22) as follows

$$
\partial_{t} \widetilde{U}_{\epsilon}+\nabla \cdot F\left(\widetilde{U}_{\epsilon}\right)-\epsilon \nu\left[\begin{array}{c}
0 \\
\triangle u_{\epsilon}
\end{array}\right]=0, \quad \text { where } \quad F\left(\widetilde{U}_{\epsilon}\right)=\left[\begin{array}{c}
\rho_{\epsilon} u_{\epsilon} \\
\rho_{\epsilon} u_{\epsilon}^{2}+p\left(\rho_{\epsilon}\right)
\end{array}\right] .
$$

Moreover, we notice that the Navier-Stokes system (5.22) in terms of entropy (4.19) has the form:

$$
\partial_{t} \eta\left(\widetilde{U}_{\epsilon}\right)+\nabla \cdot q\left(\widetilde{U}_{\epsilon}\right)-\epsilon \nu\left[\begin{array}{c}
0 \\
u_{\epsilon} \triangle u_{\epsilon}
\end{array}\right]=0, \quad \text { where } \quad q(\widetilde{U})=u_{\epsilon}\left(\eta\left(\widetilde{U}_{\epsilon}\right)+p\left(\rho_{\epsilon}\right)\right) .
$$

Let us multiply the Navier-Stokes system (5.33), from the left, by $2 \widetilde{U}_{\epsilon}^{T} \eta^{\prime \prime}\left(U_{\epsilon}\right)$

$$
2 \widetilde{U}_{\epsilon}^{T} \eta^{\prime \prime}\left(\widetilde{U}_{\epsilon}\right) \partial_{t} \widetilde{U}_{\epsilon}+2 \widetilde{U}_{\epsilon}^{T} \eta^{\prime \prime}\left(\widetilde{U}_{\epsilon}\right) F^{\prime}\left(\widetilde{U}_{\epsilon}\right) \nabla \cdot \widetilde{U}_{\epsilon}-\epsilon \nu 2 \widetilde{U}_{\epsilon}^{T} \eta^{\prime \prime}\left(\widetilde{U}_{\epsilon}\right)\left[\begin{array}{c}
0 \\
\triangle u_{\epsilon}
\end{array}\right]=0
$$

We note that

$$
\widetilde{U}_{\epsilon}^{T} \eta^{\prime \prime}\left(\widetilde{U}_{\epsilon}\right)\left[\begin{array}{c}
0 \\
\triangle u_{\epsilon}
\end{array}\right]=0
$$


and, therefore, we have

$$
2 \widetilde{U}_{\epsilon}^{T} \eta^{\prime \prime}\left(\widetilde{U}_{\epsilon}\right) \partial_{t} \widetilde{U}_{\epsilon}=\partial_{t}\left[\widetilde{U}_{\epsilon}^{T} \eta^{\prime \prime}\left(\widetilde{U}_{\epsilon}\right) \widetilde{U}_{\epsilon}\right]-2 \widetilde{U}_{\epsilon}^{T} \partial_{t} \eta^{\prime \prime}\left(\widetilde{U}_{\epsilon}\right) \widetilde{U}_{\epsilon} .
$$

Moreover, by virtue of

$$
\eta^{\prime \prime}(U) F^{\prime}(U)=\left(F^{\prime}(U)\right)^{T} \eta^{\prime \prime}(U)
$$

we find

$$
2 \widetilde{U}_{\epsilon}^{T} \eta^{\prime \prime}\left(\widetilde{U}_{\epsilon}\right) F^{\prime}\left(\widetilde{U}_{\epsilon}\right) \nabla \cdot \widetilde{U}_{\epsilon}=\nabla \cdot\left[\widetilde{U}_{\epsilon}^{T} \eta^{\prime \prime}\left(\widetilde{U}_{\epsilon}\right) F^{\prime}\left(\widetilde{U}_{\epsilon}\right) \widetilde{U}_{\epsilon}\right]-2 \widetilde{U}_{\epsilon}^{T} \nabla \cdot\left[\eta^{\prime \prime}\left(\widetilde{U}_{\epsilon}\right) F^{\prime}\left(\widetilde{U}_{\epsilon}\right)\right] \widetilde{U}_{\epsilon} .
$$

Integrating over $[0, t] \times\left\{x_{1}>0\right\}\left(x^{\prime} \in \mathbb{R}^{n-1}\right)$, we obtain

$$
\begin{gathered}
\int_{0}^{t} \int_{x_{1}>0} \partial_{t}\left[\widetilde{U}_{\epsilon}^{T} \eta^{\prime \prime}\left(\widetilde{U}_{\epsilon}\right) \widetilde{U}_{\epsilon}\right] d x d s+\int_{0}^{t} \int_{x_{1}>0} \nabla \cdot\left[\widetilde{U}_{\epsilon}^{T} \eta^{\prime \prime}\left(\widetilde{U}_{\epsilon}\right) F^{\prime}\left(\widetilde{U}_{\epsilon}\right) \widetilde{U}_{\epsilon}\right] d x d s \\
-2 \int_{0}^{t} \int_{x_{1}>0} \widetilde{U}_{\epsilon}^{T} \partial_{t} \eta^{\prime \prime}\left(\widetilde{U}_{\epsilon}\right) \widetilde{U}_{\epsilon} d x d s-2 \int_{0}^{t} \int_{x_{1}>0} \widetilde{U}_{\epsilon}^{T} \nabla \cdot\left[\eta^{\prime \prime}\left(\widetilde{U}_{\epsilon}\right) F^{\prime}\left(\widetilde{U}_{\epsilon}\right)\right] \widetilde{U}_{\epsilon} d x d s=0 .
\end{gathered}
$$

We integrate by parts:

$$
\begin{aligned}
& -2 \int_{0}^{t} \int_{x_{1}>0} \widetilde{U}_{\epsilon}^{T} \partial_{t} \eta^{\prime \prime}\left(\widetilde{U}_{\epsilon}\right) \widetilde{U}_{\epsilon} d x d s=-2 \int_{x_{1}>0} \widetilde{U}_{\epsilon}^{T} \eta^{\prime \prime}\left(\widetilde{U}_{\epsilon}\right) \widetilde{U}_{\epsilon} d x \\
& +\left.2 \int_{x_{1}>0} \widetilde{U}_{\epsilon}^{T} \eta^{\prime \prime}\left(\widetilde{U}_{\epsilon}\right) \widetilde{U}_{\epsilon}\right|_{t=0} d x+4 \int_{0}^{t} \int_{x_{1}>0} \partial_{t} \widetilde{U}_{\epsilon}^{T} \eta^{\prime \prime}\left(\widetilde{U}_{\epsilon}\right) \widetilde{U}_{\epsilon} d x d s,
\end{aligned}
$$

whence we find

$$
\begin{aligned}
& -2 \int_{0}^{t} \int_{x_{1}>0} \widetilde{U}_{\epsilon}^{T} \nabla \cdot\left[\eta^{\prime \prime}\left(\widetilde{U}_{\epsilon}\right) F^{\prime}\left(\widetilde{U}_{\epsilon}\right)\right] \widetilde{U}_{\epsilon} d x d s=-2 \int_{0}^{t} \int_{x_{1}=0} \widetilde{U}_{\epsilon}^{T} \eta^{\prime \prime}\left(\widetilde{U}_{\epsilon}\right) F^{\prime}\left(\widetilde{U}_{\epsilon}\right) \widetilde{U}_{\epsilon} d x^{\prime} d s \\
& +4 \int_{0}^{t} \int_{x_{1}>0} \nabla \cdot \widetilde{U}_{\epsilon}^{T}\left[\eta^{\prime \prime}\left(\widetilde{U}_{\epsilon}\right) F^{\prime}\left(\widetilde{U}_{\epsilon}\right)\right] \widetilde{U}_{\epsilon} d x d s .
\end{aligned}
$$

Noticing that

$$
4 \int_{0}^{t} \int_{x_{1}>0} \partial_{t} \widetilde{U}_{\epsilon}^{T} \eta^{\prime \prime}\left(\widetilde{U}_{\epsilon}\right) \widetilde{U}_{\epsilon} d x d s+\int_{0}^{t} \int_{x_{1}>0} \nabla \cdot \widetilde{U}_{\epsilon}^{T}\left[\eta^{\prime \prime}\left(\widetilde{U}_{\epsilon}\right) F^{\prime}\left(\widetilde{U}_{\epsilon}\right)\right] \widetilde{U}_{\epsilon} d x d s=0,
$$

we result in

$$
\begin{aligned}
& \int_{x_{1}>0} \widetilde{U}_{\epsilon}^{T} \eta^{\prime \prime}\left(\widetilde{U}_{\epsilon}\right) \widetilde{U}_{\epsilon} d x-\left.\int_{x_{1}>0} \widetilde{U}_{\epsilon}^{T} \eta^{\prime \prime}\left(\widetilde{U}_{\epsilon}\right) \widetilde{U}_{\epsilon}\right|_{t=0} d x \\
& -\left.\int_{0}^{t} \int_{\mathbb{R}^{n-1}} \widetilde{U}_{\epsilon}^{T} \eta^{\prime \prime}\left(\widetilde{U}_{\epsilon}\right) F^{\prime}\left(\widetilde{U}_{\epsilon}\right) \widetilde{U}_{\epsilon}\right|_{x_{1}=0} d x^{\prime} d s=0 .
\end{aligned}
$$

We recall that $\eta^{\prime \prime}\left(\widetilde{U}_{\epsilon}\right)$ is positive definite, consequently

$$
\widetilde{U}_{\epsilon}^{T} \eta^{\prime \prime}\left(\widetilde{U}_{\epsilon}\right) \widetilde{U}_{\epsilon} \geq \delta\left|\widetilde{U}_{\epsilon}\right|^{2},
$$

for some $\delta>0$. 
Therefore, we obtain for the initial data

$$
\widetilde{U}_{0}=\left[\begin{array}{c}
\rho_{0}+\epsilon I \\
\epsilon\left(\rho_{0}+\epsilon I\right)\left(\frac{c}{\rho_{0}} I+\epsilon v_{1}, \sqrt{\epsilon} \vec{w}\right)
\end{array}\right]\left(-\frac{x_{1}}{c}, \epsilon x_{1}, \sqrt{\epsilon} x^{\prime}\right)
$$

and for the first component of velocity the relation

$$
\int_{x_{1}>0} \widetilde{U}_{\epsilon}^{2} d x-\int_{x_{1}>0} \widetilde{U}_{0}^{2} d x-\left.\int_{0}^{t} \int_{\mathbb{R}^{n-1}} \widetilde{U}_{1, \epsilon}^{T} \eta^{\prime \prime}\left(\widetilde{U}_{1, \epsilon}\right) F^{\prime}\left(\widetilde{U}_{1, \epsilon}\right) \widetilde{U}_{1, \epsilon}\right|_{x_{1}=0} d x^{\prime} d s \leq 0 .
$$

Let us now consider the integral on the boundary. With notation $u_{1}=u_{1, \epsilon}$ for the first component of velocity and with $H^{\prime \prime}(\rho)=\frac{p^{\prime}(\rho)}{\rho}$, we see that

$$
\begin{aligned}
& \widetilde{U}_{1, \epsilon}^{T} \eta^{\prime \prime}\left(\widetilde{U}_{1, \epsilon}\right) F^{\prime}\left(\widetilde{U}_{1, \epsilon}\right) \widetilde{U}_{1, \epsilon} \\
= & \left(\rho_{\epsilon}, \rho_{\epsilon} u_{1}\right)^{T}\left(\begin{array}{cc}
H^{\prime \prime}\left(\rho_{\epsilon}\right)+\frac{u_{\epsilon}^{2}}{2} & -\frac{u_{1}}{\rho_{\epsilon}} \\
-\frac{u_{1}}{\rho_{\epsilon}} & \frac{1}{\rho_{\epsilon}}
\end{array}\right)\left(\begin{array}{cc}
0 & 1 \\
-u_{\epsilon}^{2}+p^{\prime}\left(\rho_{\epsilon}\right) & 2 u_{1}
\end{array}\right)\left(\begin{array}{c}
\rho_{\epsilon} \\
\rho_{\epsilon} u_{1}
\end{array}\right) \\
= & \rho_{\epsilon}^{2} u_{1}\left(H^{\prime \prime}\left(\rho_{\epsilon}\right)+\frac{u_{\epsilon}^{2}}{2}\right)-u_{1}\left(\left(-u_{\epsilon}^{2}+p^{\prime}\left(\rho_{\epsilon}\right)\right) \rho_{\epsilon}+2 \rho_{\epsilon} u_{1}^{2}\right) \\
& +\rho_{\epsilon} u_{1}\left(-u_{1}^{2}+\frac{1}{\rho_{\epsilon}}\left(\left(-u_{\epsilon}^{2}+p^{\prime}\left(\rho_{\epsilon}\right)\right) \rho_{\epsilon}+2 \rho_{\epsilon} u_{1}^{2}\right)\right)=u_{1} \rho_{\epsilon}\left(p^{\prime}\left(\rho_{\epsilon}\right)+\rho_{\epsilon} \frac{u_{\epsilon}^{2}}{2}\right)-u_{1}^{3} \rho_{\epsilon} .
\end{aligned}
$$

Let us consider the initial condition $I_{0}(t, y)$ for the KZK equation of the type found in Remark 5.6. We suppose (without loss of generality) that $I_{0}=0$ for $\left.t \in\right] 0, L[$ only once at the point $\frac{L}{2}$. More precisely, we suppose that the sign of $u_{1}$ is changing in the following way:

- $u_{1} \leq 0$ for $t \in\left[0+(k-1) L, \frac{L}{2}+(k-1) L\right](k=1,2,3, \ldots)$,

- $u_{1}>0$ for $t \in\left(\frac{L}{2}+(k-1) L, k L\right)(k=1,2,3, \ldots)$.

If $t \in\left[0, \frac{L}{2}\right]$ (for $k=1$ ), the first component of the velocity is negative

$$
\left.u_{1}\right|_{x_{1}=0}=\epsilon \frac{c}{\rho_{0}} I_{0}(t, y)+\epsilon^{2} G\left(I_{0}\right)(t, y)<0,
$$

where $G\left(I_{0}\right)$ is a $L$-periodic function with mean value zero from equation (5.28) $\left(\left.u_{1}\right|_{x_{1}=0}=0\right.$ for $t=0$, and $\left.t=\frac{L}{2}\right)$, then we have

$$
u_{1} \rho_{\epsilon}\left(p^{\prime}\left(\rho_{\epsilon}\right)+\rho_{\epsilon} \frac{u_{\epsilon}^{2}}{2}\right)-u_{1}^{3} \rho_{\epsilon}<0
$$

since the negative term is of the order of $\epsilon^{2}$ and the positive term $-u_{1}^{3} \rho_{\epsilon}$ is of the order of $\epsilon^{3}$. Therefore, for $t \in\left[0, \frac{L}{2}\right]$ we find

$$
\int_{x_{1}>0} \widetilde{U}_{\epsilon}^{2} d x \leq \int_{x_{1}>0} \widetilde{U}_{0}^{2} d x
$$

If $t \in\left(\frac{L}{2}, L\right)$, the first component of velocity is positive $\left.u_{1}\right|_{x_{1}=0}>0$, then we also impose $\left.\rho_{\epsilon}\right|_{x_{1}=0}=\rho_{0}+\epsilon I_{0}(t, y)$, where $I_{0}(t, y)$ is the initial condition for the KZK equation. Thus, the term $u_{1}^{3} \rho_{\epsilon}$ has the "correct" sign. For the term

$$
u_{1} \rho_{\epsilon}\left(p^{\prime}\left(\rho_{\epsilon}\right)+\rho_{\epsilon} \frac{u_{\epsilon}^{2}}{2}\right)>0
$$


we see that on the boundary it has the form

$$
\begin{aligned}
& \epsilon\left(\frac{c}{\rho_{0}} I_{0}+\left.\epsilon v_{1}\right|_{z=0}\right)\left(\rho_{0}+\epsilon I_{0}\right)\left(c^{2} \epsilon+\frac{(\gamma-1) c^{2}}{\rho_{0}} \epsilon^{2} I_{0}+\left(\rho_{0}+\epsilon I_{0}\right)\left(\left(\epsilon \frac{c}{\rho_{0}} I_{0}\right.\right.\right. \\
& \left.\left.\left.+\left.\epsilon^{2} v_{1}\right|_{z=0}\right)^{2}+\left.\epsilon^{3} \vec{w}^{2}\right|_{z=0}\right)\right) \leq \epsilon^{2} C_{0} I_{0}
\end{aligned}
$$

for some constant $C_{0}=c^{3}+\delta$. Consequently, we obtain

$$
\begin{aligned}
& \int_{0}^{t}\left(u_{1} \rho_{\epsilon}\left(p^{\prime}\left(\rho_{\epsilon}\right)+\rho_{\epsilon} \frac{u_{\epsilon}^{2}}{2}\right)-u_{1}^{3} \rho_{\epsilon}\right) d s=\int_{0}^{\frac{L}{2}}\left(u_{1} \rho_{\epsilon}\left(p^{\prime}\left(\rho_{\epsilon}\right)+\rho_{\epsilon} \frac{u_{\epsilon}^{2}}{2}\right)-u_{1}^{3} \rho_{\epsilon}\right) d s \\
+ & \int_{\frac{L}{2}}^{t}\left(u_{1} \rho_{\epsilon}\left(p^{\prime}\left(\rho_{\epsilon}\right)+\rho_{\epsilon} \frac{u_{\epsilon}^{2}}{2}\right)-u_{1}^{3} \rho_{\epsilon}\right) d s \\
\leq & -\left|\int_{0}^{\frac{L}{2}}\left(u_{1} \rho_{\epsilon}\left(p^{\prime}\left(\rho_{\epsilon}\right)+\rho_{\epsilon} \frac{u_{\epsilon}^{2}}{2}\right)-u_{1}^{3} \rho_{\epsilon}\right) d s\right|+\int_{\frac{L}{2}}^{L} u_{1} \rho_{\epsilon}\left(p^{\prime}\left(\rho_{\epsilon}\right)+\rho_{\epsilon} \frac{u_{\epsilon}^{2}}{2}\right) d s \\
& -\int_{\frac{L}{2}}^{L} u_{1}^{3} \rho_{\epsilon} d t \leq \epsilon^{2} C_{0} \int_{\frac{L}{2}}^{L} I_{0} d t=\epsilon^{2+\alpha} C_{0} \int_{\frac{L}{2}}^{L} \tilde{I}_{0} d t=\epsilon^{2+\alpha} \widetilde{K},
\end{aligned}
$$

where $\widetilde{K}=O(1)$ is a positive constant independent of time and $\alpha \geq 0$ is the order of "the sufficiently small" initial data $I_{0}$.

Since $\widetilde{U}_{0}=O(1)$, we obtain for all $t \leq L+\frac{L}{2}$ (for $\left.t \in\left[L, L+\frac{L}{2}\right] u_{1}\right|_{x_{1}=0}<0$ and $\left.\int_{L}^{L+\frac{L}{2}}\left(u_{1} \rho_{\epsilon}\left(p^{\prime}\left(\rho_{\epsilon}\right)+\rho_{\epsilon} \frac{u_{\epsilon}^{2}}{2}\right)-u_{1}^{3} \rho_{\epsilon}\right) d s<0\right)$ the estimate

$$
\int_{x_{1}>0} \widetilde{U}_{\epsilon}^{2} d x \leq \int_{x_{1}>0} \widetilde{U}_{0}^{2} d x+\epsilon^{2+\alpha} \widetilde{K} .
$$

However, for $t<2 L+\frac{L}{2}$ we have, due to the periodicity of $I_{0}$,

$$
\int_{x_{1}>0} \widetilde{U}_{\epsilon}^{2} d x \leq \int_{x_{1}>0} \widetilde{U}_{0}^{2} d x+2 \epsilon^{2+\alpha} \widetilde{K} .
$$

Then we conclude that for $t<\kappa L+\frac{L}{2}$

$$
\int_{x_{1}>0} \widetilde{U}_{\epsilon}^{2} d x \leq \int_{x_{1}>0} \widetilde{U}_{0}^{2} d x+\kappa \epsilon^{2+\alpha} \widetilde{K} .
$$

To keep the sense of the bounded a priori estimate we need to impose that

$$
\kappa \epsilon^{2+\alpha} \widetilde{K}=O(1), \quad \text { or } \quad \kappa<\frac{1}{\widetilde{K} \epsilon^{2+\alpha}} .
$$

Thus, for

$$
t<L\left(\frac{1}{\widetilde{K} \epsilon^{2+\alpha}}+\frac{1}{2}\right)=T
$$

or more compactly

$$
t<\frac{T_{0}}{\epsilon^{2+\alpha}}
$$


with a constant $T_{0}=O(1)$, we obtain that

$$
\widetilde{U}_{\epsilon} \in L_{\infty}(] 0, T\left[, L_{2}\left(\left\{x_{1}>0\right\} \times \mathbb{R}^{n-1}\right)\right) .
$$

If $I_{0}=0$ for $\left.t \in\right] 0, L$ [ a finite number of times $m$, and, moreover, $I_{0}$ is negative, for example, in the first interval, then we have the a priori estimate for $t<L \kappa=T$, where

$$
\kappa<\frac{T_{0}}{\left(\widetilde{K}_{1}+\ldots+\widetilde{K}_{r}\right) \epsilon^{2+\alpha}}, \quad r=\left[\frac{m+1}{2}\right] .
$$

To prove now that $\widetilde{U}_{\epsilon} \in L_{\infty}(] 0, T\left[, H^{s^{\prime}-2}\left(\left\{x_{1}>0\right\} \times \mathbb{R}^{n-1}\right)\right)$ (for $\widetilde{U}_{0} \in H^{s^{\prime}-2}\left(\left\{x_{1}>0\right\} \times \mathbb{R}^{n-1}\right)$, where $s^{\prime}$ is from the condition of Theorem 5.5), we use the result of $[10$, p.352] for incompletely parabolic problems. We also find that $\partial_{t} \widetilde{U}_{\epsilon} \in L_{\infty}(] 0, T\left[, H^{s^{\prime}-4}\left(\left\{x_{1}>0\right\} \times \mathbb{R}^{n-1}\right)\right)$.

Using the standard Faedo-Galerkin method and the theorem concerning a sequential compactness of the unit ball in the Hilbert space, we obtain the existence of the unique solution of the Navier-Stokes system. More precisely,

$$
\rho_{\epsilon} \in C\left(\left[0, T\left[, H^{s^{\prime}}\left(\left\{x_{1}>0\right\} \times \mathbb{R}^{n-1}\right)\right) \cap C^{1}\left(\left[0, T\left[, H^{s^{\prime}-2}\left(\left\{x_{1}>0\right\} \times \mathbb{R}^{n-1}\right)\right)\right.\right.\right.\right.
$$

and

$$
u_{\epsilon} \in C\left(\left[0, T\left[, H^{s^{\prime}-2}\left(\left\{x_{1}>0\right\} \times \mathbb{R}^{n-1}\right)\right) \cap C^{1}\left(\left[0, T\left[, H^{s^{\prime}-4}\left(\left\{x_{1}>0\right\} \times \mathbb{R}^{n-1}\right)\right) .\right.\right.\right.\right.
$$

5.3. Approximation result for the general viscous case. Let us construct the approximate system for the Navier-Stokes system (5.22).

We take as the state equation

$$
p=p\left(\rho_{\epsilon}\right)=c^{2} \epsilon \tilde{\rho}_{\epsilon}+\frac{(\gamma-1) c^{2}}{2 \rho_{0}} \epsilon^{2} \tilde{\rho}_{\epsilon}^{2} .
$$

Then we construct, according to the formulas, the functions $v$ from equation (4.5), $w$ from equation (4.6), and

$$
\begin{aligned}
v_{1}(\tau, z, y)= & -\frac{c^{2}}{\rho_{0}}\left(\int_{0}^{\tau} \partial_{z} I(s, z, y) d s+\int_{0}^{L} \frac{s}{L} \partial_{z} I(s, z, y) d s\right) \\
& +\frac{(\gamma-1)}{2 \rho_{0}^{2}} c I^{2}-\frac{c(\gamma-1)}{2 L \rho_{0}^{2}} \int_{0}^{L} I^{2}(\tau, z, y) d \tau+\frac{\nu}{c \rho_{0}^{2}} \partial_{\tau} I .
\end{aligned}
$$

In the above formula, the terms containing $\int_{0}^{L}$ correspond to the definition of the operator $\partial_{\tau}^{-1}$, which implies that $v_{1}$ is $L$-periodic in $\tau$ and has mean value zero. To exclude the derivative with respect to $z$ from equation (5.35) we find from the KZK equation that

$$
-\frac{c^{2}}{\rho_{0}} \partial_{\tau}^{-1} \partial_{z} I=-\frac{(\gamma+1) c}{4 \rho_{0}^{2}} I^{2}+\frac{c(\gamma+1)}{4 \rho_{0}^{2} L} \int_{0}^{L} I^{2} d s-\frac{\nu}{2 c \rho^{2}} \partial_{\tau} I-\frac{c^{3}}{2 \rho_{0}} \partial_{\tau}^{-2} \triangle_{y} I
$$

and thus we obtain relation (5.25). Next, we introduce the density and velocities (4.8)-(4.10) and construct the function $\bar{U}(4.11)$. 
The function $\bar{U}_{\epsilon} \stackrel{\text { note }}{=}\left(\bar{\rho}_{\epsilon}, \bar{u}_{\epsilon}\right)$, defined in equation (4.11) by densities and velocities from equations (4.8)-(4.10), is the solution of the problem

$$
\begin{aligned}
& \partial_{t} \bar{\rho}_{\epsilon}+\nabla \cdot\left(\bar{\rho}_{\epsilon} \bar{u}_{\epsilon}\right) \\
&= \epsilon^{3}\left(\rho_{0} \partial_{z} v_{1}+\partial_{z}(I v)-\frac{1}{c} \partial_{\tau}\left(I v_{1}\right)+\nabla_{y}(I w)\right)+\epsilon^{4} \partial_{z}\left(I v_{1}\right), \\
& \bar{\rho}_{\epsilon}\left(\partial_{t} \bar{u}_{\epsilon, 1}+\left(\bar{u}_{\epsilon} \cdot \nabla\right) \bar{u}_{\epsilon, 1}\right)+\partial_{x_{1}} p\left(\bar{\rho}_{\epsilon}\right)-\epsilon \nu \Delta \bar{u}_{\epsilon, 1} \\
&=\epsilon^{3}\left(I \partial_{\tau} v_{1}-\frac{1}{2 c} I \partial_{\tau} v^{2}+\frac{\rho_{0}}{2} \partial_{z} v^{2}-\frac{\rho_{0}}{c} \partial_{\tau}\left(v v_{1}\right)+\rho_{0} w \nabla_{y} v\right. \\
&\left.+\frac{(\gamma-1)}{2 \rho_{0}} c^{2} \partial_{z} I^{2}+\frac{2 \nu}{c} \partial_{z \tau}^{2} v-\nu \Delta_{y} v-\frac{\nu}{c^{2}} \partial_{\tau}^{2} v_{1}\right) \\
&+\epsilon^{4}\left(\frac{I}{2} \partial_{z} v^{2}-\frac{1}{c} I \partial_{\tau}\left(v v_{1}\right)+I w \nabla_{y} v+\rho_{0} \partial_{z}\left(v v_{1}\right)-\frac{\rho_{0}}{2 c} \partial_{\tau} v_{1}^{2}\right. \\
&\left.+\rho_{0} w \nabla_{y} v_{1}-\nu \partial_{z}^{2} v+\frac{2 \nu}{c} \partial_{z \tau}^{2} v_{1}-\nu \Delta_{y} v_{1}\right) \\
&+\epsilon^{5}\left(I \partial_{z}\left(v v_{1}\right)-\frac{1}{2 c} I \partial_{\tau} v_{1}^{2}+I w \nabla_{y} v_{1}+\frac{\rho_{0}}{2} \partial_{z} v_{1}^{2}-\nu \partial_{z}^{2} v_{1}\right)+\epsilon^{6} I v_{1} \partial_{z} v_{1}, \\
&+\epsilon^{\frac{11}{2}}\left(I v_{1} \partial_{z} w\right) \cdot \\
&+\epsilon^{\frac{7}{2}}\left(\rho_{0} v \partial_{z} w-\frac{\rho_{0}}{c} v_{1} \partial_{\tau} w+\frac{\rho_{0}}{2} \nabla_{y} w^{2}-\frac{I}{c} v \partial_{\tau} w-\nu \Delta w+\frac{2 \nu}{c} \partial_{z \tau}^{2} w\right) \\
&+\bar{\rho}_{\epsilon}\left(\partial_{t} \bar{u}_{\epsilon}^{\prime}+\left(u_{\epsilon} \partial_{z} w+\nabla\right) \bar{u}_{\epsilon}^{\prime}\right)+\partial_{x^{\prime}} p\left(\bar{\rho}_{\epsilon}\right)-\epsilon \nu \Delta \bar{u}_{\epsilon}^{\prime} \\
&+\frac{\gamma}{2}\left(\rho_{0} c^{2} \nabla_{y} I^{2}-\frac{\nu}{c} \partial_{\tau}^{2} \vec{w}\right)
\end{aligned}
$$

To control the terms in right-hand sides, we need that

$$
\partial_{z}^{3} I \in L_{\infty}\left(\left[0, \infty\left[, L_{2}(\tau \times y)\right),\right.\right.
$$

therefore, in theorem 2.1 we take $s>\max \left\{6,\left[\frac{n}{2}\right]+1\right\}$. Then the remaining terms of equations (5.36)-(5.38) are bounded in $L_{2}$.

Thus, we have two systems: system (5.22) and

$$
\partial_{t} \bar{\rho}_{\epsilon}+\operatorname{div}\left(\bar{\rho}_{\epsilon} \bar{u}_{\epsilon}\right)=\epsilon^{\frac{5}{2}} R_{1}, \quad \bar{\rho}_{\epsilon}\left[\partial_{t} \bar{u}_{\epsilon}+\left(\bar{u}_{\epsilon} \cdot \nabla\right) \bar{u}_{\epsilon}\right]+\nabla p\left(\bar{\rho}_{\epsilon}\right)-\epsilon \nu \Delta \bar{u}_{\epsilon}=\epsilon^{\frac{5}{2}} \vec{R}_{2} .
$$

By $\epsilon^{\frac{5}{2}} R_{1}=O\left(\epsilon^{3}\right)$ we denote the remaining term of the first equation of the NavierStokes system (5.36). 
The second equation of the Navier-Stokes system (5.39) has the remaining term $\epsilon^{\frac{5}{2}} \vec{R}_{2}=\left(O\left(\epsilon^{3}\right), O\left(\epsilon^{\frac{5}{2}}\right)\right)=O\left(\epsilon^{\frac{5}{2}}\right)$, presented by equation (5.37) in the $x_{1}$-direction and by equation $(5.38)$ in the $x^{\prime}$-direction.

THEOREM 5.7. Let us suppose that the assumptions of the Theorem 5.5 hold and that there exists a unique global solution in time $\bar{U}_{\epsilon}=\left(\bar{\rho}_{\epsilon}, \bar{u}_{\epsilon}\right)$ of the approximate system (5.36)-(5.38) deduced from a solution of the KZK equation with the help of equations (4.5), (4.6), (5.25). The function $\bar{U}_{\epsilon}\left(x_{1}, x^{\prime}, t\right)=\bar{U}_{\epsilon}\left(x_{1}-c t, \epsilon x_{1}, \sqrt{\epsilon} x^{\prime}\right)$, given by formula (4.11), is defined in the half space (5.24). Moreover, according to its definition, we have (5.29) and (5.30).

Then there exists a constant $C>0$ such that for all small enough $\epsilon$ the solutions $\left(\rho_{\epsilon}, u_{\epsilon}\right)$ of system (5.22) and $\left(\bar{\rho}_{\epsilon}, \bar{u}_{\epsilon}\right)$ of system (5.36)-(5.38) satisfy the following stability estimate

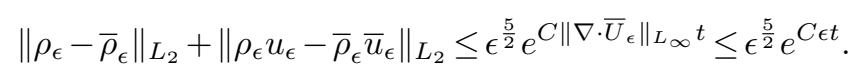

Estimate (5.40) ensures that its left-hand side remains smaller than order $\epsilon$ for any finite time

$$
0<t<\frac{T}{\epsilon} \ln \frac{1}{\epsilon}
$$

where $T$ is a positive constant and $T=O(1)$.

Proof. We have two systems (see (5.33) and (5.34))

$$
\begin{aligned}
& \left\{\begin{array}{l}
\partial_{t} \eta\left(\widetilde{U}_{\epsilon}\right)+\nabla \cdot q\left(\widetilde{U}_{\epsilon}\right)-\epsilon \nu\left[\begin{array}{c}
0 \\
u_{\epsilon} \triangle u_{\epsilon}
\end{array}\right]=0, \\
\partial_{t} \widetilde{U}_{\epsilon}+\nabla \cdot F\left(\widetilde{U}_{\epsilon}\right)-\epsilon \nu\left[\begin{array}{c}
0 \\
\triangle u_{\epsilon}
\end{array}\right]=0
\end{array}\right. \\
& \left\{\begin{array}{l}
\partial_{t} \eta\left(\bar{U}_{\epsilon}\right)+\nabla \cdot q\left(\bar{U}_{\epsilon}\right)-\epsilon \nu\left[\begin{array}{c}
0 \\
\bar{u}_{\epsilon} \triangle \bar{u}_{\epsilon}
\end{array}\right]=\epsilon^{\frac{5}{2}}\left(\frac{\eta\left(\bar{U}_{\epsilon}\right)+p\left(\bar{\rho}_{\epsilon}\right)}{\bar{\rho}_{\epsilon}} R_{1}+\bar{u}_{\epsilon} \vec{R}_{2}\right), \\
\partial_{t} \bar{U}_{\epsilon}+\nabla \cdot F\left(\bar{U}_{\epsilon}\right)-\epsilon \nu\left[\begin{array}{c}
0 \\
\triangle \bar{u}_{\epsilon}
\end{array}\right]=\epsilon^{\frac{5}{2}} \vec{R},
\end{array}\right.
\end{aligned}
$$

where $\vec{R}=\left(R_{1}, \overrightarrow{R_{2}}\right)$ is the remaining term from system (5.39). Since we suppose that $\bar{U}_{\epsilon}=\left(\bar{\rho}_{\epsilon}, \bar{\rho}_{\epsilon} \bar{u}_{\epsilon}\right)^{T}$ is bounded we can again denote

$$
\bar{R}=\frac{\eta\left(\bar{U}_{\epsilon}\right)+p\left(\bar{\rho}_{\epsilon}\right)}{\bar{\rho}_{\epsilon}} R_{1}+\bar{u}_{\epsilon} \vec{R}_{2}
$$

Let us compute

$$
\partial_{t}\left(\eta\left(\widetilde{U}_{\epsilon}\right)-\eta\left(\bar{U}_{\epsilon}\right)-\eta^{\prime}\left(\bar{U}_{\epsilon}\right)\left(\widetilde{U}_{\epsilon}-\bar{U}_{\epsilon}\right)\right) .
$$

First we find from initial systems that

$$
\partial_{t}\left(\eta\left(\widetilde{U}_{\epsilon}\right)-\eta\left(\bar{U}_{\epsilon}\right)\right)=-\nabla \cdot\left(\left(q\left(\widetilde{U}_{\epsilon}\right)-q\left(\bar{U}_{\epsilon}\right)\right)+\epsilon \nu\left[\begin{array}{c}
0 \\
u_{\epsilon} \triangle u_{\epsilon}-\bar{u}_{\epsilon} \triangle \bar{u}_{\epsilon}
\end{array}\right]-\epsilon^{\frac{5}{2}} \bar{R} .\right.
$$

Therefore, we notice that

$$
-\partial_{t}\left(\eta^{\prime}\left(\bar{U}_{\epsilon}\right)\left(\widetilde{U}_{\epsilon}-\bar{U}_{\epsilon}\right)\right)=-\left(\partial_{t} \bar{U}_{\epsilon}\right)^{T} \eta^{\prime \prime}\left(\bar{U}_{\epsilon}\right)\left(\widetilde{U}_{\epsilon}-\bar{U}_{\epsilon}\right)-\eta^{\prime}\left(\bar{U}_{\epsilon}\right)\left(\partial_{t} \widetilde{U}_{\epsilon}-\partial_{t} \bar{U}_{\epsilon}\right),
$$


where

$$
\left(\partial_{t} \bar{U}_{\epsilon}\right)^{T} \eta^{\prime \prime}\left(\bar{U}_{\epsilon}\right)\left(\widetilde{U}_{\epsilon}-\bar{U}_{\epsilon}\right)=\left(-\nabla \cdot F\left(\bar{U}_{\epsilon}\right)+\left[\begin{array}{c}
0 \\
\epsilon \nu \triangle \bar{u}_{\epsilon}
\end{array}\right]+\epsilon^{\frac{5}{2}} \vec{R}\right)^{T} \eta^{\prime \prime}\left(\bar{U}_{\epsilon}\right)\left(\widetilde{U}_{\epsilon}-\bar{U}_{\epsilon}\right),
$$

and

$$
\begin{aligned}
-\eta^{\prime}\left(\bar{U}_{\epsilon}\right)\left(\partial_{t} \widetilde{U}_{\epsilon}-\partial_{t} \bar{U}_{\epsilon}\right)= & -\eta^{\prime}\left(\bar{U}_{\epsilon}\right)\left(-\nabla \cdot F\left(\widetilde{U}_{\epsilon}\right)+\nabla \cdot F\left(\bar{U}_{\epsilon}\right)\right) \\
& -\eta^{\prime}\left(\bar{U}_{\epsilon}\right) \epsilon \nu\left[\begin{array}{c}
0 \\
\triangle u_{\epsilon}-\triangle \bar{u}_{\epsilon}
\end{array}\right]+\epsilon^{\frac{5}{2}} \eta^{\prime}\left(\bar{U}_{\epsilon}\right) \vec{R} .
\end{aligned}
$$

Using the property for the convex entropy

$$
\eta^{\prime \prime}(U) F^{\prime}(U)=\left(F^{\prime}(U)\right)^{T} \eta^{\prime \prime}(U),
$$

we find

$$
\begin{aligned}
& \left(\nabla \cdot F\left(\bar{U}_{\epsilon}\right)\right)^{T} \eta^{\prime \prime}\left(\bar{U}_{\epsilon}\right)\left(\widetilde{U}_{\epsilon}-\bar{U}_{\epsilon}\right)=\nabla \cdot \bar{U}_{\epsilon}^{T}\left(F^{\prime}\left(\bar{U}_{\epsilon}\right)\right)^{T} \eta^{\prime \prime}\left(\bar{U}_{\epsilon}\right)\left(\widetilde{U}_{\epsilon}-\bar{U}_{\epsilon}\right) \\
= & \nabla \cdot \bar{U}_{\epsilon}^{T} \eta^{\prime \prime}\left(\bar{U}_{\epsilon}\right) F^{\prime}\left(\bar{U}_{\epsilon}\right)\left(\widetilde{U}_{\epsilon}-\bar{U}_{\epsilon}\right) .
\end{aligned}
$$

Thus, we obtain

$$
\begin{aligned}
& \partial_{t}\left(\eta\left(\widetilde{U}_{\epsilon}\right)-\eta\left(\bar{U}_{\epsilon}\right)-\eta^{\prime}\left(\bar{U}_{\epsilon}\right)\left(\widetilde{U}_{\epsilon}-\bar{U}_{\epsilon}\right)\right)=-\nabla \cdot\left(q\left(\widetilde{U}_{\epsilon}\right)-q\left(\bar{U}_{\epsilon}\right)\right) \\
& \quad+\epsilon \nu\left[\begin{array}{c}
0 \\
u_{\epsilon} \triangle u_{\epsilon}-\bar{u}_{\epsilon} \triangle \bar{u}_{\epsilon}
\end{array}\right]-\epsilon^{\frac{5}{2}} \bar{R}+\nabla \cdot \bar{U}_{\epsilon}^{T} \eta^{\prime \prime}\left(\bar{U}_{\epsilon}\right) F^{\prime}\left(\bar{U}_{\epsilon}\right)\left(\widetilde{U}_{\epsilon}-\bar{U}_{\epsilon}\right) \\
& -\left[\begin{array}{c}
0 \\
\epsilon \nu \triangle \bar{u}_{\epsilon}
\end{array}\right]^{T} \eta^{\prime \prime}\left(\bar{U}_{\epsilon}\right)\left(\widetilde{U}_{\epsilon}-\bar{U}_{\epsilon}\right)-\epsilon^{\frac{5}{2}} \vec{R}^{T} \eta^{\prime \prime}\left(\bar{U}_{\epsilon}\right)\left(\widetilde{U}_{\epsilon}-\bar{U}_{\epsilon}\right)+\epsilon^{\frac{5}{2}} \eta^{\prime}\left(\bar{U}_{\epsilon}\right) \vec{R} \\
& -\eta^{\prime}\left(\bar{U}_{\epsilon}\right)\left(-\nabla \cdot F\left(\widetilde{U}_{\epsilon}\right)+\nabla \cdot F\left(\bar{U}_{\epsilon}\right)\right)-\eta^{\prime}\left(\bar{U}_{\epsilon}\right) \epsilon \nu\left[\begin{array}{c}
0 \\
\triangle u_{\epsilon}-\triangle \bar{u}_{\epsilon}
\end{array}\right]
\end{aligned}
$$

where, due to equations (5.31) and (5.32) (and $\left.\frac{\rho}{\bar{\rho}_{\epsilon}}=s+1\right)$,

$$
\begin{aligned}
& -\left[\begin{array}{c}
0 \\
\epsilon \nu \triangle \bar{u}_{\epsilon}
\end{array}\right]^{T} \eta^{\prime \prime}\left(\bar{U}_{\epsilon}\right)\left(\widetilde{U}_{\epsilon}-\bar{U}_{\epsilon}\right)=-\epsilon \nu\left[\begin{array}{c}
-\frac{\bar{u}_{\epsilon}}{\overline{\bar{\rho}}_{\epsilon}} \triangle \bar{u}_{\epsilon} \\
\frac{1}{\bar{\rho}_{\epsilon}} \triangle \bar{u}_{\epsilon}
\end{array}\right]^{T}\left[\begin{array}{c}
\rho_{\epsilon}-\bar{\rho}_{\epsilon} \\
\rho u-\bar{\rho}_{\epsilon} \bar{u}_{\epsilon}
\end{array}\right] \\
= & -\epsilon \nu \triangle \bar{u}_{\epsilon}\left(-\bar{u}_{\epsilon}, 1\right)\left[\begin{array}{c}
s \\
u(s+1)-\bar{u}_{\epsilon}
\end{array}\right]=-\epsilon \nu \triangle \bar{u}_{\epsilon}\left(u_{\epsilon}-\bar{u}_{\epsilon}\right) \\
& -\epsilon \nu \triangle \bar{u}_{\epsilon} \frac{\rho_{\epsilon}-\bar{\rho}_{\epsilon}}{\bar{\rho}_{\epsilon}}\left(u_{\epsilon}-\bar{u}_{\epsilon}\right)
\end{aligned}
$$

and

$$
-\eta^{\prime}\left(\bar{U}_{\epsilon}\right) \epsilon \nu\left[\begin{array}{c}
0 \\
\triangle u_{\epsilon}-\triangle \bar{u}_{\epsilon}
\end{array}\right]=-\epsilon \nu \bar{u}_{\epsilon}\left(\triangle u_{\epsilon}-\triangle \bar{u}_{\epsilon}\right) .
$$

We integrate equation (5.41) over the half space (5.24). The integration by parts gives, with notations $q_{1}, F_{1}$ for first components of vectors $q$ and $F$,

$$
\begin{aligned}
& \frac{d}{d t} \int_{x_{1}>0}\left(\eta\left(\widetilde{U}_{\epsilon}\right)-\eta\left(\bar{U}_{\epsilon}\right)-\eta^{\prime}\left(\bar{U}_{\epsilon}\right)\left(\widetilde{U}_{\epsilon}-\bar{U}_{\epsilon}\right) d x\right. \\
= & -\int_{x_{1}=0}\left(q_{1}\left(\widetilde{U}_{\epsilon}\right)-q_{1}\left(\bar{U}_{\epsilon}\right)-\eta^{\prime}\left(\bar{U}_{\epsilon}\right)^{T}\left(F_{1}\left(\widetilde{U}_{\epsilon}\right)-F_{1}\left(\bar{U}_{\epsilon}\right)\right)\right) d x^{\prime} \\
& -\int_{x_{1}>0} \nabla \cdot \bar{U}_{\epsilon}^{T} \eta^{\prime \prime}\left(\bar{U}_{\epsilon}\right)\left(F\left(\widetilde{U}_{\epsilon}\right)-F\left(\bar{U}_{\epsilon}\right)-F^{\prime}\left(\bar{U}_{\epsilon}\right)\left(\widetilde{U}_{\epsilon}-\bar{U}_{\epsilon}\right)\right) d x
\end{aligned}
$$




$$
\begin{aligned}
& +\epsilon \nu \int_{x_{1}=0}\left(u_{\epsilon} \partial_{x_{1}} u_{\epsilon}-\bar{u}_{\epsilon} \partial_{x_{1}} \bar{u}_{\epsilon}\right) d x^{\prime}-\epsilon \nu \int_{x_{1}>0}\left(\left|\nabla \cdot u_{\epsilon}\right|^{2}-\left|\nabla \cdot \bar{u}_{\epsilon}\right|^{2}\right) d x \\
& -\epsilon^{\frac{5}{2}} \int_{x_{1}>0}\left(\bar{R}-\eta^{\prime}\left(\bar{U}_{\epsilon}\right) \vec{R}\right) d x-\epsilon^{\frac{5}{2}} \int_{x_{1}>0} \vec{R}^{T} \eta^{\prime \prime}\left(\bar{U}_{\epsilon}\right)\left(\widetilde{U}_{\epsilon}-\bar{U}_{\epsilon}\right) d x \\
& +\epsilon \nu \int_{x_{1}>0} \triangle \bar{u}_{\epsilon} \frac{\rho_{\epsilon}-\bar{\rho}_{\epsilon}}{\bar{\rho}_{\epsilon}}\left(u_{\epsilon}-\bar{u}_{\epsilon}\right) d x+\epsilon \nu \int_{x_{1}=0}\left(-\bar{u}_{\epsilon} \partial_{x_{1}}\left(u_{\epsilon}-\bar{u}_{\epsilon}\right)-\partial_{x_{1}} \bar{u}_{\epsilon}\left(u_{\epsilon}-\bar{u}_{\epsilon}\right)\right) d x^{\prime} \\
& +\epsilon \nu \int_{x_{1}>0}\left(\nabla \cdot \bar{u}_{\epsilon} \cdot \nabla \cdot\left(u_{\epsilon}-\bar{u}_{\epsilon}\right)+\nabla \cdot \bar{u}_{\epsilon} \cdot \nabla \cdot\left(u_{\epsilon}-\bar{u}_{\epsilon}\right)\right) d x
\end{aligned}
$$

It is easy to see that

$$
\begin{aligned}
& \epsilon \nu \int_{x_{1}=0}\left(u_{\epsilon} \partial_{x_{1}} u_{\epsilon}-\bar{u}_{\epsilon} \partial_{x_{1}} \bar{u}_{\epsilon}\right) d x^{\prime}+\epsilon \nu \int_{x_{1}=0}\left(-\bar{u}_{\epsilon} \partial_{x_{1}}\left(u_{\epsilon}-\bar{u}_{\epsilon}\right)\right. \\
& \left.-\partial_{x_{1}} \bar{u}_{\epsilon}\left(u_{\epsilon}-\bar{u}_{\epsilon}\right)\right) d x^{\prime}=\epsilon \nu \int_{x_{1}=0}\left(u_{\epsilon}-\bar{u}_{\epsilon}\right) \partial_{x_{1}}\left(u_{\epsilon}-\bar{u}_{\epsilon}\right) d x^{\prime}
\end{aligned}
$$

and

$$
\begin{aligned}
& -\epsilon \nu \int_{x_{1}>0}\left(\left|\nabla \cdot u_{\epsilon}\right|^{2}-\left|\nabla \cdot \bar{u}_{\epsilon}\right|^{2}\right) d x+\epsilon \nu \int_{x_{1}>0}\left(\nabla \cdot \bar{u}_{\epsilon} \cdot \nabla \cdot\left(u_{\epsilon}-\bar{u}_{\epsilon}\right)\right. \\
& \left.+\nabla \cdot \bar{u}_{\epsilon} \cdot \nabla \cdot\left(u_{\epsilon}-\bar{u}_{\epsilon}\right)\right) d x=-\epsilon \nu \int_{x_{1}>0}\left|\nabla \cdot\left(u_{\epsilon}-\bar{u}_{\epsilon}\right)\right|^{2} d x .
\end{aligned}
$$

Let us now consider the boundary condition

$$
\begin{aligned}
& -\int_{x_{1}=0}\left(q_{1}\left(\widetilde{U}_{\epsilon}\right)-q_{1}\left(\bar{U}_{\epsilon}\right)-\eta^{\prime}\left(\bar{U}_{\epsilon}\right)^{T}\left(F_{1}\left(\widetilde{U}_{\epsilon}\right)-F_{1}\left(\bar{U}_{\epsilon}\right)\right)\right) d x^{\prime} \\
= & \left.\left(q_{1}\left(\widetilde{U}_{\epsilon}\right)-q_{1}\left(\bar{U}_{\epsilon}\right)-\eta^{\prime}\left(\bar{U}_{\epsilon}\right)^{T}\left(F_{1}\left(\widetilde{U}_{\epsilon}\right)-F_{1}\left(\bar{U}_{\epsilon}\right)\right)\right)\right|_{x_{1}=0 .}
\end{aligned}
$$

Equation (5.44) takes the explicit form:

$$
\begin{aligned}
& \left.\left(q_{1}\left(\widetilde{U}_{\epsilon}\right)-q_{1}\left(\bar{U}_{\epsilon}\right)-\eta^{\prime}\left(\bar{U}_{\epsilon}\right)^{T}\left(F_{1}\left(\widetilde{U}_{\epsilon}\right)-F_{1}\left(\bar{U}_{\epsilon}\right)\right)\right)\right|_{x_{1}=0}=u_{1}\left(\eta\left(\widetilde{U}_{\epsilon}\right)+p\left(\rho_{\epsilon}\right)\right) \\
& -\bar{u}_{1}\left(\eta\left(\bar{U}_{\epsilon}\right)+p\left(\bar{\rho}_{\epsilon}\right)\right)-\left.\left[\begin{array}{c}
H^{\prime}\left(\bar{\rho}_{\epsilon}\right)-\frac{\bar{u}_{\epsilon}^{2}}{2} \\
\bar{u}_{1}
\end{array}\right]^{T}\left[\begin{array}{c}
\rho_{\epsilon} u_{1}-\bar{\rho}_{\epsilon} \bar{u}_{1} \\
\rho_{\epsilon} u_{\epsilon}^{2}+p\left(\rho_{\epsilon}\right)-\bar{\rho}_{\epsilon} \bar{u}_{\epsilon}^{2}-p\left(\bar{\rho}_{\epsilon}\right)
\end{array}\right]\right|_{x_{1}=0} .
\end{aligned}
$$

Choosing $\left.u_{1}\right|_{x_{1}=0}=\left.\bar{u}_{1}\right|_{x_{1}=0}$, we use the convexity of the entropy $\eta$,

$$
\eta\left(\widetilde{U}_{\epsilon}\right)-\eta\left(\bar{U}_{\epsilon}\right)-\eta^{\prime}\left(\bar{U}_{\epsilon}\right)\left(\widetilde{U}_{\epsilon}-\bar{U}_{\epsilon}\right) \geq \alpha\left|\widetilde{U}_{\epsilon}-\bar{U}_{\epsilon}\right|^{2},
$$

to obtain

$$
\begin{aligned}
& \left.\left(q_{1}\left(\widetilde{U}_{\epsilon}\right)-q_{1}\left(\bar{U}_{\epsilon}\right)-\eta^{\prime}\left(\bar{U}_{\epsilon}\right)^{T}\left(F_{1}\left(\widetilde{U}_{\epsilon}\right)-F_{1}\left(\bar{U}_{\epsilon}\right)\right)\right)\right|_{x_{1}=0} \\
= & u_{1}\left(\eta\left(\widetilde{U}_{\epsilon}\right)-\eta\left(\bar{U}_{\epsilon}\right)+p\left(\rho_{\epsilon}\right)-p\left(\bar{\rho}_{\epsilon}\right)\right) \\
& -u_{1}\left(\rho_{\epsilon}-\bar{\rho}_{\epsilon}\right)\left(H^{\prime}\left(\bar{\rho}_{\epsilon}\right)-\frac{\bar{u}_{\epsilon}^{2}}{2}\right)-\left.u_{1}\left[\rho_{\epsilon} u_{\epsilon}^{2}-\bar{\rho}_{\epsilon} \bar{u}_{\epsilon}^{2}+p\left(\rho_{\epsilon}\right)-p\left(\bar{\rho}_{\epsilon}\right)\right]\right|_{x_{1}=0} \\
= & \left.u_{1}\left(\eta\left(\widetilde{U}_{\epsilon}\right)-\eta\left(\bar{U}_{\epsilon}\right)-\eta^{\prime}\left(\bar{U}_{\epsilon}\right)^{T}\left(\widetilde{U}_{\epsilon}-\bar{U}_{\epsilon}\right)\right)\right|_{x_{1}=0} .
\end{aligned}
$$

Therefore, boundary condition (5.45) takes the same sign as the first component of velocity $\left.u_{1}\right|_{x_{1}=0}$ because

$$
\eta\left(\widetilde{U}_{\epsilon}\right)-\eta\left(\bar{U}_{\epsilon}\right)-\eta^{\prime}\left(\bar{U}_{\epsilon}\right)^{T}\left(\widetilde{U}_{\epsilon}-\bar{U}_{\epsilon}\right) \geq\left(\widetilde{U}_{\epsilon}-\bar{U}_{\epsilon}\right)^{2} \geq 0
$$


is always positive.

Thus, for the boundary conditions we take $\left.u_{\epsilon}\right|_{x_{1}=0}=\left.\bar{u}_{\epsilon}\right|_{x_{1}=0}$ and if $u_{1}>0$, moreover, we also suppose $\left.\rho_{\epsilon}\right|_{x_{1}=0}=\left.\bar{\rho}_{\epsilon}\right|_{x_{1}=0}$. Then the first boundary condition (5.43) is always equal to zero

$$
\epsilon \nu \int_{x_{1}=0}\left(u_{\epsilon}-\bar{u}_{\epsilon}\right) \partial_{x_{1}}\left(u_{\epsilon}-\bar{u}_{\epsilon}\right) d x^{\prime}=-\left.\epsilon \nu\left(u_{\epsilon}-\bar{u}_{\epsilon}\right) \partial_{x_{1}}\left(u_{\epsilon}-\bar{u}_{\epsilon}\right)\right|_{x_{1}=0}=0 .
$$

For the second boundary condition (5.45), if $\left.u_{\epsilon}\right|_{x_{1}=0}<0$ then we have in the lefthand side of the estimate a positive quantity on the boundary which can be omitted.

If $\left.u_{\epsilon}\right|_{x_{1}=0}>0$ then we have for $\left.\rho_{\epsilon}\right|_{x_{1}=0}=\left.\bar{\rho}_{\epsilon}\right|_{x_{1}=0}$

$$
\begin{aligned}
& \left.u_{1}\left(H\left(\rho_{\epsilon}\right)+\rho_{\epsilon} \frac{u_{\epsilon}^{2}}{2}-H\left(\bar{\rho}_{\epsilon}\right)-\bar{\rho}_{\epsilon} \frac{\bar{u}_{\epsilon}^{2}}{2}-\left(\rho_{\epsilon}-\bar{\rho}_{\epsilon}\right)\left(H^{\prime}\left(\bar{\rho}_{\epsilon}\right)-\frac{\bar{u}_{\epsilon}^{2}}{2}\right)-\rho_{\epsilon} u_{\epsilon}^{2}+\bar{\rho}_{\epsilon} \bar{u}_{\epsilon}^{2}\right)\right|_{x_{1}=0} \\
= & \left.u_{1}\left(H\left(\rho_{\epsilon}\right)-H\left(\bar{\rho}_{\epsilon}\right)-\left(\rho_{\epsilon}-\bar{\rho}_{\epsilon}\right) H^{\prime}\left(\bar{\rho}_{\epsilon}\right)+\rho_{\epsilon}\left(\frac{\bar{u}_{\epsilon}^{2}}{2}-\frac{u_{\epsilon}^{2}}{2}\right)\right)\right|_{x_{1}=0} \\
= & \left.u_{1}\left(H\left(\rho_{\epsilon}\right)-H\left(\bar{\rho}_{\epsilon}\right)-\left(\rho_{\epsilon}-\bar{\rho}_{\epsilon}\right) H^{\prime}\left(\bar{\rho}_{\epsilon}\right)\right)\right|_{x_{1}=0}=0 .
\end{aligned}
$$

We use the Taylor expansion

$$
F\left(\widetilde{U}_{\epsilon}\right)-F\left(\bar{U}_{\epsilon}\right)=F^{\prime}\left(\bar{U}_{\epsilon}\right)\left(\widetilde{U}_{\epsilon}-\bar{U}_{\epsilon}\right)+O\left(\left|\widetilde{U}_{\epsilon}-\bar{U}_{\epsilon}\right|^{2}\right),
$$

and we have

$$
F\left(\widetilde{U}_{\epsilon}\right)-F\left(\bar{U}_{\epsilon}\right)-F^{\prime}\left(\bar{U}_{\epsilon}\right)\left(\widetilde{U}_{\epsilon}-\bar{U}_{\epsilon}\right) \leq C\left|\widetilde{U}_{\epsilon}-\bar{U}_{\epsilon}\right|^{2} .
$$

Taking the same initial data $\left.\bar{U}_{\epsilon}\right|_{t=0}=\left.\widetilde{U}_{\epsilon}\right|_{t=0}$, we obtain

$$
\frac{d}{d t} \int_{x_{1}>0}\left|\widetilde{U}_{\epsilon}-\bar{U}_{\epsilon}\right|^{2} d x \leq C\left\|\nabla \cdot \bar{U}_{\epsilon}\right\|_{L_{\infty}} \int_{x_{1}>0}\left|\widetilde{U}_{\epsilon}-\bar{U}_{\epsilon}\right|^{2} d x+K \epsilon^{5} .
$$

Here the constants $K$ and $C$ do not depend on $t$.

Therefore, applying the Gronwall lemma and recalling that $\nabla \cdot \bar{U}_{\epsilon}=O(\epsilon)$, we have

$$
\left\|\widetilde{U}_{\epsilon}-\bar{U}_{\epsilon}\right\|_{L_{2}\left(\left\{x_{1}>0\right\}\right)}^{2} \leq K \epsilon^{5} e^{\epsilon C t} .
$$

As the difference of the solutions $\widetilde{U}_{\epsilon}-\bar{U}_{\epsilon}$ is of the order of $O(\epsilon)$ and the left-hand side of estimation (5.46) is of the order of $O\left(\epsilon^{2}\right)$, our goal is to have the inequality

$$
\epsilon^{5} \int_{0}^{t} e^{C \epsilon(t-s)} d s<\epsilon^{2}
$$

From $\epsilon^{2} e^{C \epsilon t}<1$ we obtain that the left-hand side of (5.40) is smaller than $\epsilon^{2}$ for time $t<T \frac{1}{\epsilon} \ln \frac{1}{\epsilon}$.

REMARK 5.8. To obtain estimate (5.40) it is sufficient to have an admissible weak solution of the Navier-Stokes system (5.22) satisfying the boundary conditions in the half space (5.24).

DeFINITION 5.9. The pair of functions $(\rho, u)$ is called an admissible weak solution of the Navier-Stokes system (5.22) satisfying the boundary conditions in the half space (5.24) if it satisfies the following properties: 
1. The pair $(\rho, u)$ is a weak solution of (5.22).

2. The pair $(\rho, u)$ satisfies in the sense of distributions (see [6, p.52])

$$
\partial_{t} \eta\left(U_{\epsilon}\right)+\nabla \cdot q\left(U_{\epsilon}\right)-\epsilon \nu\left[\begin{array}{c}
0 \\
u_{\epsilon} \triangle u_{\epsilon}
\end{array}\right] \leq 0,
$$

or equivalently, for any positive twice differentiable test function $\psi$ with compact support in the half space (5.24) $(\rho, u)$ satisfies

$$
\begin{aligned}
& \int_{0}^{T} \int_{x_{1}>0}\left(\partial_{t} \psi \eta\left(U_{\epsilon}\right)+\nabla \cdot \psi q\left(U_{\epsilon}\right)-\epsilon \nu\left[\begin{array}{c}
0 \\
\left.0 \cdot u_{\epsilon}\right|^{2}
\end{array}\right] \psi+\epsilon \nu\left[\begin{array}{c}
0 \\
\nabla \frac{u_{\epsilon}^{2}}{2}
\end{array}\right] \nabla \cdot \psi\right) d x d t \\
& +\int_{x_{1}>0} \psi(x, 0) \eta\left(U_{0}(x)\right) d x \\
& +\left.\int_{0}^{T} \int_{\mathbb{R}^{n-1}} \psi\left(q\left(U_{\epsilon}\right)-\epsilon \nu\left[\begin{array}{c}
0 \\
u_{\epsilon} \cdot \nabla \cdot u_{\epsilon}
\end{array}\right]\right)\right|_{x_{1}=0} d x^{\prime} d t \geq 0 .
\end{aligned}
$$

3. The pair $(\rho, u)$ satisfies the equality

$$
\begin{aligned}
& -\int_{x_{1}>0} \frac{U_{\epsilon}^{2}}{2} d x+\int_{0}^{t} \int_{x_{1}>0}\left(\nabla \cdot U_{\epsilon} F\left(U_{\epsilon}\right)+\epsilon \nu\left[\begin{array}{c}
0 \\
\left.\nabla \cdot u_{\epsilon}\right|^{2}
\end{array}\right]\right) d x d s \\
& +\int_{x_{1}>0} U_{0}^{2}(x) d x+\left.\int_{0}^{t} \int_{\mathbb{R}^{n-1}} U_{\epsilon}\left(F\left(U_{\epsilon}\right)-\epsilon \nu\left[\begin{array}{c}
0 \\
\nabla u_{\epsilon}
\end{array}\right]\right)\right|_{x_{1}=0} d x^{\prime} d t=0 .
\end{aligned}
$$

We obtain as a consequence the following theorem.

THEOREM 5.10. To obtain estimate (5.40) it is sufficient to have an admissible weak solution of the Navier-Stokes system (5.22)

$$
\partial_{t} U_{\epsilon}+\nabla \cdot F\left(U_{\epsilon}\right)-\epsilon \nu\left[\begin{array}{c}
0 \\
\triangle u_{\epsilon}
\end{array}\right]=0
$$

satisfying the boundary conditions in the half space (5.24), such that [17, 21] $\rho_{\epsilon} \geq 0$ a.e.,

$$
\begin{aligned}
& \rho_{\epsilon} \in L_{\infty}\left((0, T), L_{2}\right) \cap C\left([0, T], L_{p}\right) \quad \text { for } \quad 1 \leq p \leq 2, \\
& \nabla \cdot u_{\epsilon} \in L_{2}\left((0, T) ; L_{2}\right), \quad \rho_{\epsilon}\left|u_{\epsilon}\right|^{2} \in L_{\infty}\left((0, T), L_{1}\right), \\
& u_{\epsilon} \in L_{2}\left((0, T), H_{0}^{1}\right), \quad \rho_{\epsilon} u_{\epsilon} \in C\left([0, T], L_{\frac{4}{3}}-\omega\right),
\end{aligned}
$$

where by $C\left([0, T], L_{\frac{4}{3}}-\omega\right)$ is denoted the space of continuous functions with values in a closed ball of $L_{\frac{4}{3}}$ endowed with the weak topology.

\section{Conclusion}

The derivation of the KZK equation in studied in the framework of nonlinear acoustic. We have introduced a corrector-function $\epsilon^{2} v_{1}$ in the physical ansatz which allows us to establish that the KZK equation is an approximation of the Euler or Navier-Stokes system. The approximation for the nonlinear KZK equation

$$
\left\|\bar{U}_{\epsilon}-U_{\epsilon}\right\|_{L_{2}}^{2} \leq \epsilon^{5} e^{C \epsilon t}
$$

is valid in the viscous and non-viscous cases. The obtained estimate guarantees that the difference $\bar{U}_{\epsilon}-U_{\epsilon}$ stays of the order of $O(\epsilon)$ during a time of the order of $\frac{1}{\epsilon} \ln \frac{1}{\epsilon}$ 
TABLE 5.1. Approximation results

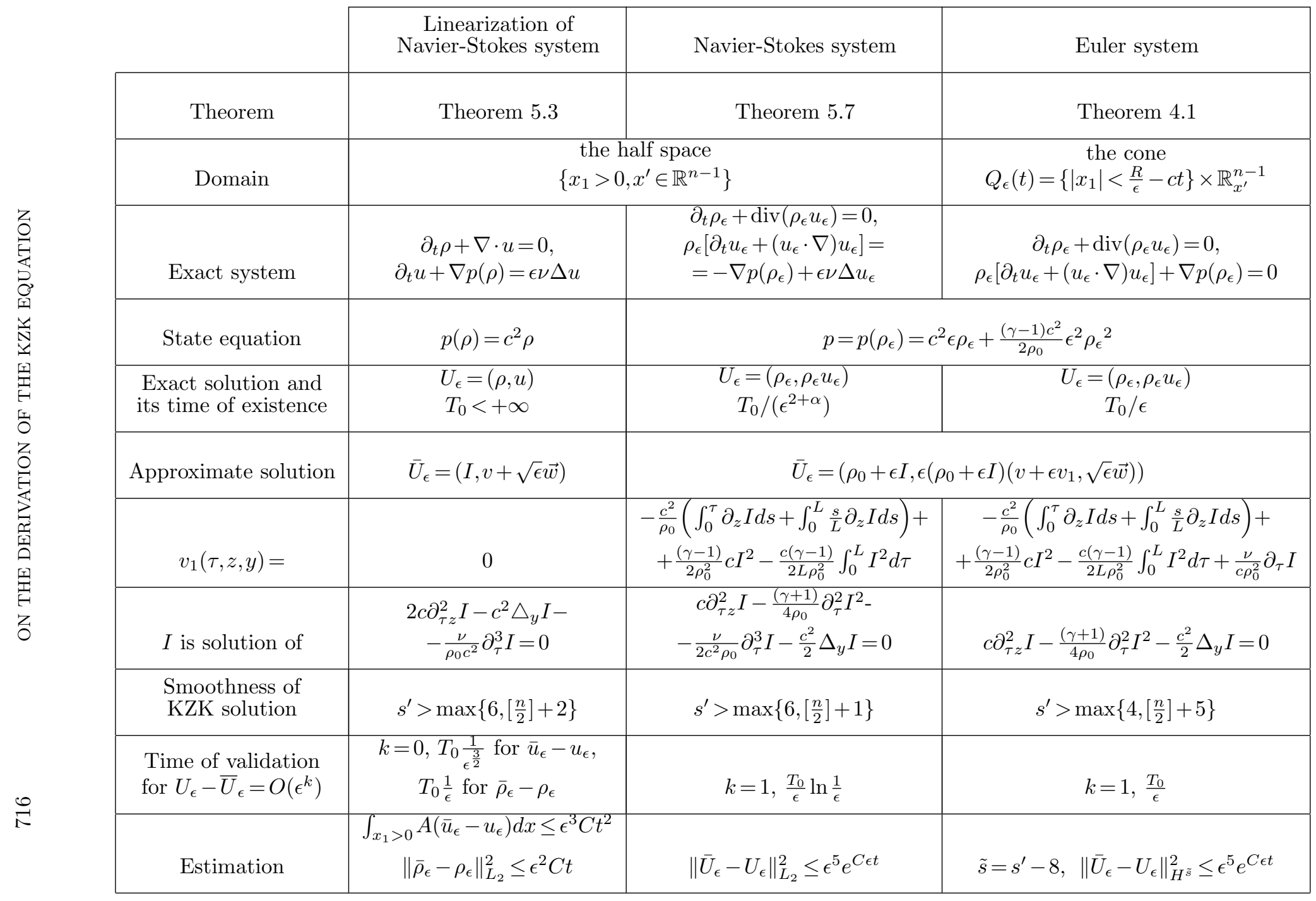


for the approximation of the Navier-Stokes system and for all existence time $\left(t<\frac{T_{0}}{\epsilon}\right)$ for the approximation of the Euler system.

Table 5.1 summarizes the approximation results, where

$$
A(u-v) \equiv\left|\partial_{t}(u-v)\right|^{2}+|\nabla \cdot(u-v)|^{2} .
$$

All approximation results are based on the regularity of the initial data for the KZK equation, which make regular the initial and boundary conditions for the Euler or Navier-Stokes system. But Remark 5.8 and Theorem 5.10 in the viscous case show that the KZK-approximation in the non-viscous case can probably hold for much less regular initial conditions. We also note that in many physical articles we can find that the initial data of the KZK equation $I_{0}$ has the form $\exp \left[-(y)^{2}\right] \sin \left(w_{0} \tau\right)$ and therefore $I_{0} \in C^{\infty}$.

Acknowledgment. I would like to thank F. Golse and J. Rauch for helpful discussions and useful suggestions, and especially C. Bardos, my scientific adviser. This work has been conducted in the laboratory Jacques Louis Lions (Paris 6).

\section{REFERENCES}

[1] S.I. Aanonsen and al., Distortion and harmonic generation in the nearfield of finite amplitude sound beam, J. Acoust. Soc. Am., 75, 749-768, 1984.

[2] M.A. Averkiou and R.O. Cleveland, Time domain numerical modeling of an electrohydraulic lithotripter with the KZK equation, J. Acoust. Soc. Am., 106, 102-112, 1999.

[3] M.A. Averkiou, Y.S. Lee and M.F. Hamilton, Self-demodulation of amplitude- and frequencymodulated pulses in a thermo-viscous fluid, J. Acoust. Soc. Am., 94, 2876-2883, 1993.

[4] M.A. Averkiou and M.F. Hamilton, Nonlinear distortion of short pulses radiated by plane and focused circular pistons, J. Acoust. Soc. Am., 102, 2539-2548, 1997.

[5] N.S. Bakhvalov, Ya. M. Zhileikin and E.A. Zabolotskaya, Nonlinear Theory of Sound Beams, American Institute of Physics, New York, 1987, Nelineinaya Teoriya Zvukovih Puchkov, Moscow "Nauka", 1982.

6] C. Dafermos, Hyperbolic Conservation Laws in Continuum Physics, Springer-Verlag, 325, 2000.

[7] P. Donnat, J.L. Joly, G. Metivier and J. Rauch, Diffractive nonlinear geometric optics, in Séminaire Équations aux Dérivées Partielles, École Polytechnique, XVII 1-23, 1995-1996, available at http://www.math.lsa.umich.edu/ rauch

[8] P. Donnat, J.L. Joly, G. Metivier and J. Rauch, Diffractive nonlinear geometric optics with rectification, Indiana Univ. Math. J., 47, 1167-1241, 1998.

[9] K.O. Friedrichs and P.D. Lax, Systems of conservation equations with a convex extension, Proc. Natl. Acad. Sci. USA, 68, 1686-1688, 1971.

[10] B. Gustafsson and A. Sundström, Incompletely parabolic problems in fluid dynamics, SIAM J. Appl. Math., 35, 343-357, 1978.

[11] S.M. Howard and C.I. Zanelli, HIFU Transducer characterization using a robust needle hydrophone, International Symposium on Therapeutic Ultrasound edited by C.C. Coussios and G. ter Haar, American Institute of Physics, 2007.

[12] T.K. Awagishi, S. Saito and Yo. Mine, Numerical calculation and measurement of nonlinear acoustic fields in ultrasound diagnosis, Jpn. J. Appl. Phys., 41, 3549-3554, 2002.

[13] R.J. Iório, W.V.L. Nunes, On equations of KP-type, Proc. Roy. Soc. Edinburgh, 128A, 725-743, 1998.

[14] A. Kitkauskaite and A. Kopustinskas, Nonlinear acoustics caused of medium, available at http://www.etf.rtu.lv/Latvieshu\%20lapa/pasn_str/konf/p_7.pdf

[15] D. Kourtiche, L. Ait Ali, L. Alliés, M. Nadi and A .Chitnalah, Harmonic propagation of finiteamplitude sound beams: second harmonic imaging in ultrasonic reflection tomography, Meas. Sci. Technol., 15, 21-28, 2004.

[16] Y.S. Lee and M.F. Hamilton, Time-domain modeling of pulsed finite-amplitude sound beams, J. Acoust. Soc. Am., 97, 906-917, 1995.

[17] P.L. Lions, Mathematical Topics in Fluid Mechanics, 2, Compressible Models, Oxford Science Publications, 1998.

[18] Ya. Li and J.A. Zagzebski, Computer model for harmonic ultrasound imaging, IEEE Tr. on Ultrasonics, Ferroelectrics, and Frequency Control, 47, 5, 1259-1272, 2000. 
[19] X. Liu, J. Lia, X. Gonga and D. Zhanga, Nonlinear absorption in biological tissue for high intensity focused ultrasound, Ultrasonics, 44, 1, e27-e30, 2006.

[20] J.A. McAteer, M.R. Bailey, J.C. Williams Jr, R.O. Cleveland and A.P. Evan, Strategies for improved shock wave lithotripsy, Minerva Urol Nefrol., 57, 4, 271-287, 2005.

[21] S. Novo, Compressible Navier-Stokes model with inflow-outflow boundary conditions, J. Math. Fluid Mech., 7, 485-514, 2005.

[22] G.F. Pinton and G.E. Trahey, Shock wave propagation with the KZK equation, IEEE Ultrasonics Symposium, 1847-1850, 2006.

[23] A. Rozanova, Khokhlov-Zabolotskaya-Kuznetsov equation, C. R. Acad. Sci. Paris, Ser. I, 344, 337-342, 2007.

[24] A. Rozanova-Pierrat, Qualitative analysis of the Khokhlov-Zabolotskaya-Kuznetsov (KZK) equation, Math. Mod. Meth. Appl. Sci., 18, 781-812, 2008.

[25] D. Sanchez, Long waves in ferromagnetic media, Zabolotskaya-Khokhlov equation, J. Diff. Equ., 210, 263-289, 2005

[26] Joshua E. Soneson and Matthew R. Myers, Gaussian representation of high-intensity focused ultrasound beams, J. Acoust. Soc. Am., 122, 5, 2526-2531, 2007.

[27] T. Sideris, Formation of Singularities in Three-dimensional compressible fluids, Commun. Math. Phys., 101, 475-485, 1985.

[28] B. Texier, The short-wave limit for nonlinear, symmetric, hyperbolic systems, Adv. Diff. Equ., 9, 1-52, 2004.

[29] M.W. Wong, An introduction to pseudo-differential operators, World Scientific, 1991.

[30] E.A. Zabolotskaya and R.V. Khokhlov, Quasi-planes waves in the nonlinear acoustic of confined beams, Sov. Phys. Acoust., 15, 35-40, 1969.

[31] D. Zhang, X.F. Gong and B. Zhang, Second harmonic sound field after insertion of a biological tissue sample (L), J. Acoust. Soc. Am., 111, 1, 45-48, 2002. 